\title{
Large Rapidity Gap Method to Select Soft Diffraction Dissociation at the LHC
}

\author{
Emily Nurse ${ }^{1}$ and Sercan Sen ${ }^{2,3}$ \\ ${ }^{1}$ Department of Physics and Astronomy, University College London, London, UK \\ ${ }^{2}$ Department of Physics Engineering, Hacettepe University, Ankara, Turkey \\ ${ }^{3}$ Department of Physics and Astronomy, University of Iowa, Iowa City, IA, USA \\ Correspondence should be addressed to Sercan Sen; Sercan.Sen@cern.ch
}

Received 27 March 2016; Revised 8 July 2016; Accepted 11 August 2016

Academic Editor: Adrian Buzatu

Copyright (C) 2016 E. Nurse and S. Sen. This is an open access article distributed under the Creative Commons Attribution License, which permits unrestricted use, distribution, and reproduction in any medium, provided the original work is properly cited. The publication of this article was funded by $\mathrm{SCOAP}^{3}$.

\begin{abstract}
In proton-proton ( $\mathrm{pp}$ ) collisions, any process involves exchanging the vacuum quantum numbers is known as diffractive process. A diffractive process with no large $Q^{2}$ is called soft diffractive process. The diffractive processes are important for understanding nonperturbative QCD effects and they also constitute a significant fraction of the total pp cross section. The diffractive events are typically characterized by a region of the detector without particles, known as a rapidity gap. In order to observe diffractive events in this way, we consider the pseudorapidity acceptance in the forward region of the ATLAS and CMS detectors at the Large Hadron Collider (LHC) and discuss the methods to select soft diffractive dissociation for pp collisions at $\sqrt{s}=7 \mathrm{TeV}$. It is shown that, in the limited detector rapidity acceptance, it is possible to select diffractive dissociation events by requiring a rapidity gap in the event; however, without using forward detectors, it seems not possible to fully separate single and double diffractive dissociation events. The Zero Degree Calorimeters can be used to distinguish the type of the diffractive processes up to a certain extent.
\end{abstract}

\section{Introduction}

The measurement of the main characteristics of diffractive interactions is essential to improve our understanding of pp collisions. However, the modelling of diffraction is still mainly generator dependent and there is no unique, agreed upon experimental definition of diffraction $[1,2]$.

While the physics of diffractive dissociation at the LHC are very important, the detector capabilities in the forward region are limited. In this paper, using the rapidity gap technique and by considering the forward rapidity coverage of the LHC experiments ATLAS [3] and CMS [4], a number of studies are carried out to select soft diffraction dissociation. In addition, the potential of Zero Degree Calorimeter for diffractive events selection is discussed.

\section{Event Classification}

In pp (or more generally hadron-hadron) scattering, interactions can be classified as either elastic or inelastic by the characteristic signatures of the final states. Furthermore, it is conventional to divide inelastic processes into diffractive and nondiffractive parts. In the theoretical concept, hadronic diffractive dissociation is principally explained to be mediated by the exchange of the Pomeron, which carries the quantum numbers of the vacuum; thus, the initial and final states in the scattering process have the same quantum numbers. If the Pomeron exchange process is additionally associated with a hard scattering (such as the production of jets, $b$-quark, and $W$ boson), the process is known as hard diffractive; otherwise, it is soft diffractive dissociation. Introductory reviews on this can be found in $[5,6]$. Diffractive events at hadron colliders can be classified into the following categories: single, double diffractive dissociation, and central diffraction (a.k.a. "Double Pomeron Exchange"), with higher order "multiPomeron" processes [7]. Thus, the total pp cross section can be written as the following series:

$$
\begin{aligned}
\sigma_{\text {total }} & =\sigma_{\text {elastic }}+\sigma_{\text {inelastic }}=\sigma_{\text {elastic }}+\sigma_{\mathrm{ND}}+\sigma_{\text {diffractive }} \\
& =\sigma_{\text {elastic }}+\sigma_{\mathrm{ND}}+\sigma_{\mathrm{SDD}}+\sigma_{\mathrm{DDD}}+\sigma_{\mathrm{DPE}}+\sigma_{\mathrm{MPE}}
\end{aligned}
$$



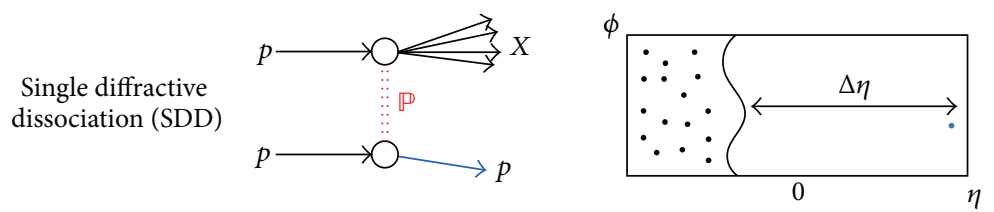

(a)
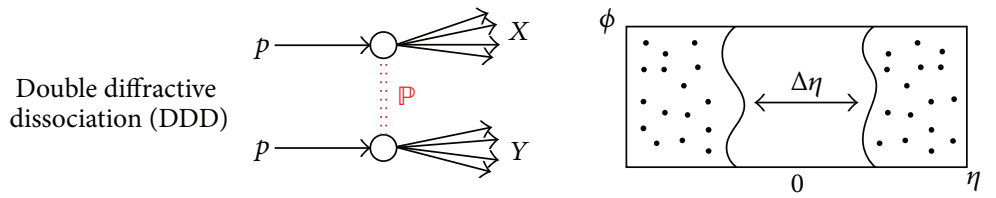

(b)

FIGURE 1: Illustration of a single (top) and double (bottom) diffractive dissociative event in which a Pomeron ( $\mathbb{P}$ ) is exchanged in a pp collision. $M_{X}$ and $M_{Y}$ are the invariant masses of the dissociation systems $X$ and $Y$, respectively. In single diffractive dissociation, $M_{Y}=m_{p}$, where $m_{p}$ is the mass of the intact proton. $\Delta \eta$ refers to the size of the large rapidity gap.

where ND is nondiffractive process, SDD (DDD) is single (double) diffraction dissociation, DPE corresponds to the Double Pomeron Exchange and MPE refers to the MultiPomeron Exchange. The precise measurements of the total cross sections of each process separately are important as they provide input to phenomenological models and are needed for tuning of the parameters of Monte Carlo event generators. At $7 \mathrm{TeV}$, diffractive processes together with the elastic scattering represent about $50 \%$ of the total pp cross section $[8,9]$.

In single diffractive dissociation, one of the incoming protons dissociates into a "low-mass" system (a system of particles with low invariant mass with respect to the centre of mass energy of the collision) while in double diffractive dissociation both of the incoming protons dissociate into "low-mass" systems as represented in Figure 1.

Diffractive events are classified by a large gap in the pseudorapidity distribution of final state particles. (the pseudorapidity of a particle is defined as $\eta=\ln \tan (\theta / 2)$, where $\theta$ is the polar angle with respect to the beam direction ( $z$-axis), and rapidity is $y=(1 / 2) \ln \left[\left(E+p_{z}\right) /\left(E-p_{z}\right)\right]$, where $p_{z}$ is the longitudinal momentum of the particle. Pseudorapidity and rapidity are equal for massless particles) The large rapidity gap can be defined as the difference between the rapidity of the diffractively scattered proton and that of the particle closest to it in (pseudo)rapidity. However, the existing ATLAS and CMS detectors are not well suited for measuring the forward rapidity gaps. Therefore, from the experimental point of view, rapidity gaps should be defined by a total absence of particles in a particular interval of pseudorapidity. The large rapidity gap, $\Delta \eta$, is the largest rapidity gap between those rapidity gaps in a final state and determines the type of the diffraction process.

\section{Diffractive Kinematical Variables}

3.1. Fractional Longitudinal Momentum Loss. In single diffractive collisions, one of the two incident protons emits a Pomeron and remains intact by losing a few percent of its initial longitudinal momentum. The fractional longitudinal momentum loss of the intact proton is related to the momentum fraction taken by the Pomeron:

$$
\xi_{X}=1-\left(\frac{p_{z}^{\text {final }}}{p_{z}^{\text {initial }}}\right),
$$

where $p_{z}^{\text {final }}$ is the final and $p_{z}^{\text {initial }}$ is the initial longitudinal momentum of the proton. The Pomeron scatters with the other beam proton and the proton dissociates into a system of particles with low invariant mass, $M_{X}$. DDD processes are described by the invariant masses $M_{X}$ and $M_{Y}$ of the dissociation systems $X$ and $Y$, respectively, as shown in Figure 1. The fractional longitudinal momentum loss of the proton can be determined by measuring the invariant mass of the dissociation system(s):

$$
\begin{gathered}
\xi_{X}=\frac{M_{X}^{2}}{s}, \\
\xi_{Y}=\frac{M_{Y}^{2}}{s},
\end{gathered}
$$

where $\sqrt{s}$ is the centre-of-mass energy for pp collisions. In the following, the convention $M_{X}>M_{Y}$ is adopted and $\xi_{X}$ is referred to as $\xi$.

3.2. Diffractive Mass. The mass of the diffractive system, $M_{X}$, can be measured experimentally by summing up the masses of all final state particles in the dissociation system:

$$
M_{X}=\sum_{i} m_{i}
$$

However, it is not possible to make a precise measurement throughout the whole pseudorapidity range due to the lack of the detector coverage in the very forward regions $[10$, 11]. Therefore, one can expect some differences between the measured mass of the diffractive system and the actual mass. Without their forward detectors, the nominal rapidity coverage of ATLAS and CMS experiments is $|\eta|<5.2$. 


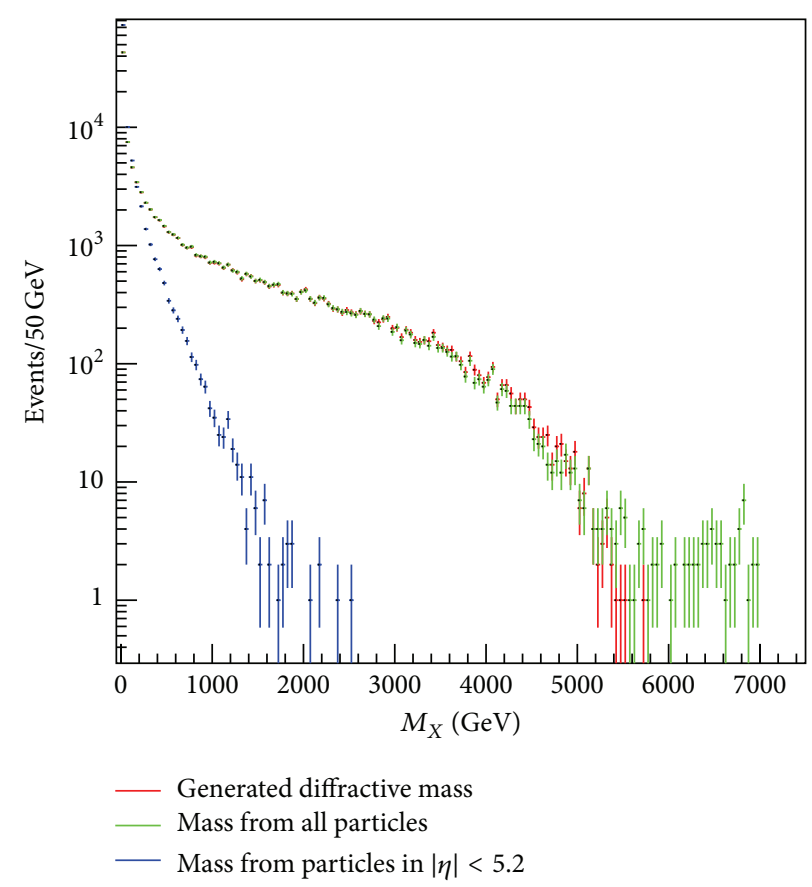

FIGURE 2: Distribution of the diffractive mass for SDD events simulated by PYTHIA 8.142. Generated diffractive mass (red line) and calculated diffractive mass from all particles in the full $\eta$ coverage (green line) and in a limited $\eta$ coverage $|\eta|<5.2$ (blue line).

The difference between the diffractive masses reconstructed from particles in $|\eta|<5.2$ and in full phase space domain is shown in Figure 2 for single diffractive events simulated by PYTHIA 8.142 [12]. It seems not possible to reconstruct the actual mass of the diffractive system within the limited rapidity range due to the particles which scatter into the very forward rapidities and escape detection. It is clear that the wider the range of rapidity covered is, the more accurately the diffractive mass can be determined.

3.3. Large Rapidity Gap. The gap signature in diffractive dissociation has been observed in the previous hadronhadron collision experiments $[13,14]$. The type of diffractive processes can be determined by looking at the number of large rapidity gaps and at their position in the rapidity space. Single diffractive dissociation processes are characterized by an edge (forward) gap only at one side of the detector while the double diffractive dissociation processes are characterized by a central gap in the central pseudorapidity region of the detector.

The large rapidity gap in an event and $\xi$ variable are closely related to each other. In SDD case, the pseudorapidity difference between the intact proton and the $X$ system is given as $\Delta \eta \sim-\ln \xi$. If the size of the large rapidity gap or the invariant mass of the dissociation system is measured, the fractional longitudinal momentum loss of the proton can be determined.

\section{Measuring Diffractive Events}

The measurements of the diffractive processes can be done based on the determination of the size of the large rapidity gap, $\Delta \eta$, and the correlation between $\Delta \eta$ and $\xi$ can be used. However, due to the forward acceptance limitations, it is also not possible to measure the gaps in the very forward rapidities or the whole size of the actual gap in some cases. It is therefore important to study the kinematical variables of the diffractive processes within the detector limits where the experimental measurements will be performed. Figure 3 shows the relation between the size of the large rapidity gap $\Delta \eta$ and $\log _{10} \xi$ for single diffractive dissociation events. As can be seen from the figure, after applying the detector acceptance cuts, the correlation between $\Delta \eta$ and $\log _{10} \xi$ is still present.

In this paper, the relation $\xi=M_{X}^{2} / s$ is used for the calculation of $\xi$. First, the largest gap in an event is reconstructed in full phase space domain. All the particles with the pseudorapidity less than (or equal to) the lower boundary of the gap are considered in one system and the rest are in the other. Then, the four vectors of all particles in the given system are summed to get the invariant mass and thus $\xi$. The RIVET [15] analysis toolkit was used throughout the analyses in this study.

4.1. Detector Rapidity Acceptance. The edge gap differential cross section, $d \sigma / d \Delta \eta$, for single and double diffractive dissociation events in the different detector rapidity acceptances $|\eta|<5.2$ and $|\eta|<8.1$ is given in Figure 4 . The distributions are normalized by the cross section of the processes obtained from PYTHIA 8. These cross sections for different processes are given in Table 1. The minimum bias event class corresponds to total inelastic collisions. As can be seen in Figure 4, the large rapidity gap distribution for SDD and DDD events is slightly different for the different 


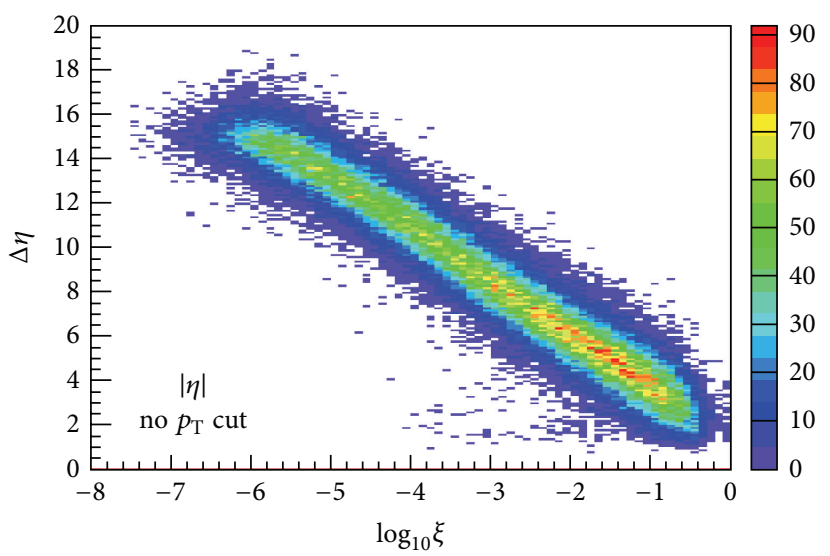

(a)

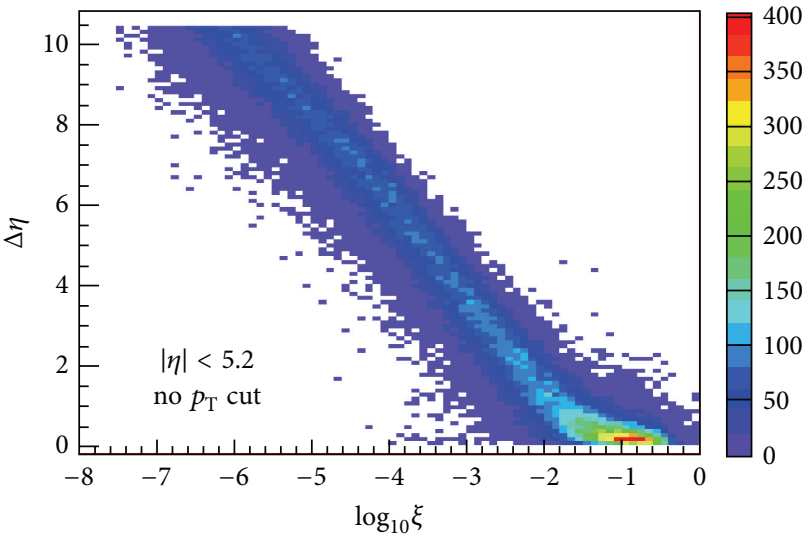

(b)

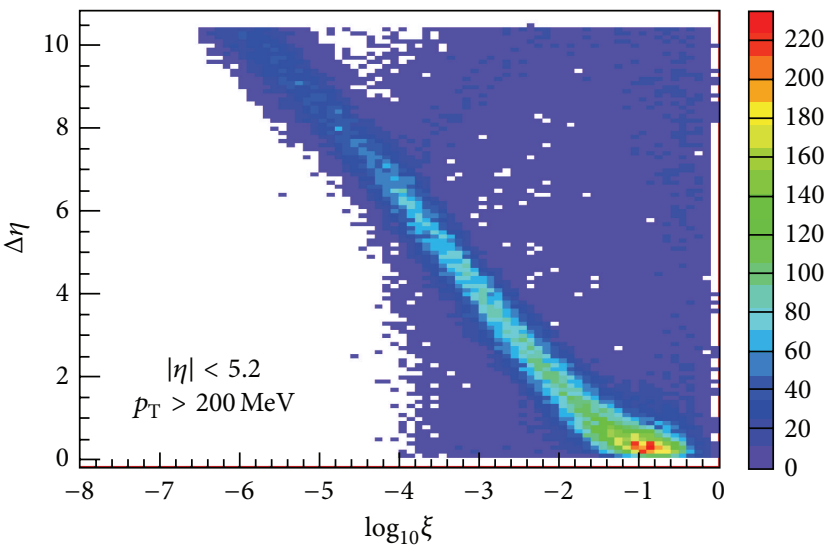

(c)

FIGURE 3: The relation between the size of the large rapidity gap, $\Delta \eta$, and $\log _{10} \xi$ for SDD events. The gap is defined as an edge gap. (a) Whole pseudorapidity range is used without any transverse momentum $\left(p_{\mathrm{T}}\right)$ threshold on particles. (b) For particles within $|\eta|<5.2$ without any $p_{\mathrm{T}}$ threshold. (c) For particles within $|\eta|<5.2$ with $p_{\mathrm{T}}>200 \mathrm{MeV}$.

TABLE 1: PYTHIA 8 cross sections at $\sqrt{s}=7 \mathrm{TeV}$.

\begin{tabular}{lc}
\hline Event class & Cross section $(\sigma[\mathrm{mb}])$ \\
\hline Single diffractive dissociation (SDD) & 13.7 \\
Double diffractive dissociation (DDD) & 9.3 \\
Diffractive dissociation (SDD + DDD) & 22.9 \\
Nondiffractive (ND) & 48.5 \\
\hline Minimum bias (SDD + DDD + ND) & 71.4 \\
\hline
\end{tabular}

detector rapidity acceptance. A clear distinction between SDD and DDD processes is possible within the larger detector acceptance $|\eta|<8.1$, but in the limited acceptance it is not possible. For the rest of this analysis, $|\eta|<5.2$ is used.

4.2. Low- $p_{\mathrm{T}}$ Threshold. It is important to make a precise measurement of the size of the large rapidity gap, since it is directly related to the mass of the dissociation system and the longitudinal momentum loss of the proton. There are several factors such as radiation from multiple partonparton interactions and accelerator related radiation that can affect the measurement. Also, limitations of detector response and resolution and the electronic noise will not allow the measurement of very low transverse momentum $\left(p_{\mathrm{T}}\right)$ particles. All these factors should be considered when using the method of large rapidity gaps for the measurement of diffractive dissociation events. In Figure 5, edge gap distributions are given for minimum bias events with various low- $p_{\mathrm{T}}$ thresholds on the final state particles. It is obvious that when the threshold is increased, some of the soft particles (i.e., pions) could have lower $p_{\mathrm{T}}$ than the threshold; therefore, the gap size becomes larger.

In addition, the distributions of $d \sigma / d \Delta \eta$ and $d \sigma / d \log _{10} \xi$ are given for different $p_{\mathrm{T}}$ cuts in Figure 6 for edge gaps and in Figure 7 for central gaps. As is clearly represented in the figures, the gap size and the ND contribution in minimum bias event content become larger with increasing $p_{\mathrm{T}}$ cut. A cut of $p_{\mathrm{T}}>500 \mathrm{MeV}$ for all final state particles enhances the size of the gap for ND events. On the other hand, each experiment must determine a reasonable $p_{\mathrm{T}}$ threshold regarding the capabilities of their detector and a low $p_{\mathrm{T}}$ cut, such as $100 \mathrm{MeV}$, might not be suitable for the measurements with the data due to the detector noise at this level. Therefore, 


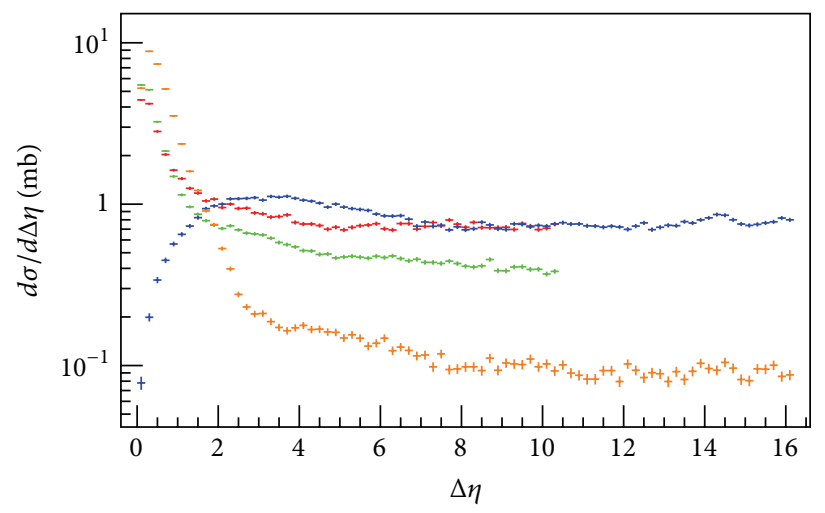

Single diffractive, $|\eta|<5.2 \quad$ Double diffractive, $|\eta|<5.2$
Single diffractive, $|\eta|<8.1 \quad$ Double diffractive, $|\eta|<8.1$

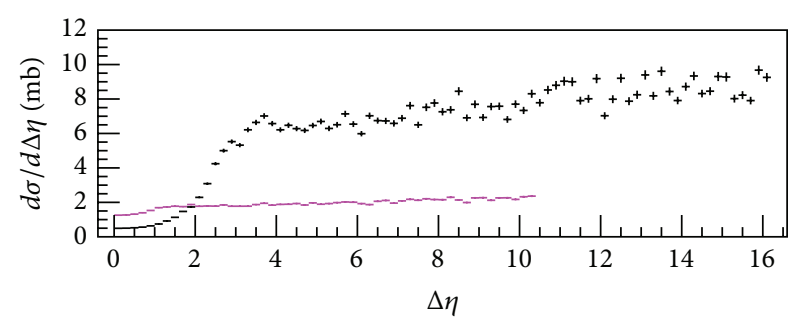

SDD/DDD, $|\eta|<5.2$

$-\mathrm{SDD} / \mathrm{DDD},|\eta|<8.1$

FigURE 4: Large rapidity gap distribution for single and double diffractive dissociation events in the different detector rapidity acceptance. The gap is defined as an edge gap. Ratio of single to double diffractive dissociation events is given on the ratio plot.

a cut of $p_{\mathrm{T}}>200 \mathrm{MeV}$ for all final state particles seems to be an ideal cut to perform the measurements experimentally. The $\Delta \eta>3$ cut is for edge gaps; for central gaps it looks like $\Delta \eta>4$ is a better cut. When $\Delta \eta>3$ cut is applied with a cut of $p_{\mathrm{T}}>200 \mathrm{MeV}$ for all final state particles in $|\eta|<5.2$, it seems possible to suppress a large fraction of ND events and select the diffractive dissociation events in minimum bias data. These cuts also will allow us to perform the measurements experimentally.

4.3. Distinguishing SDD and DDD Events. From a phenomenological point of view, looking at the number and position of the large rapidity gaps in rapidity space, one can differentiate the type of the diffractive process. However, considering the limited rapidity coverage of the detectors, this might not be possible since the gap sizes in the very forward rapidities are not measured precisely.

4.3.1. Edge Gaps. The distinguishability of SDD and DDD events in $|\eta|<5.2$ is studied by requiring an edge gap in the events. The visible cross sections of events that pass the various $\Delta \eta$ cuts with $p_{\mathrm{T}}>200 \mathrm{MeV}$ for all final state particles are given in Table 2. Similarly, the visible cross sections for different cuts on $p_{\mathrm{T}}$ of the final state particles for $\Delta \eta>3$ are given in Table 3. The cross section for ND events is higher for high $p_{\mathrm{T}}$ cuts. On the other hand, ND events are suppressed

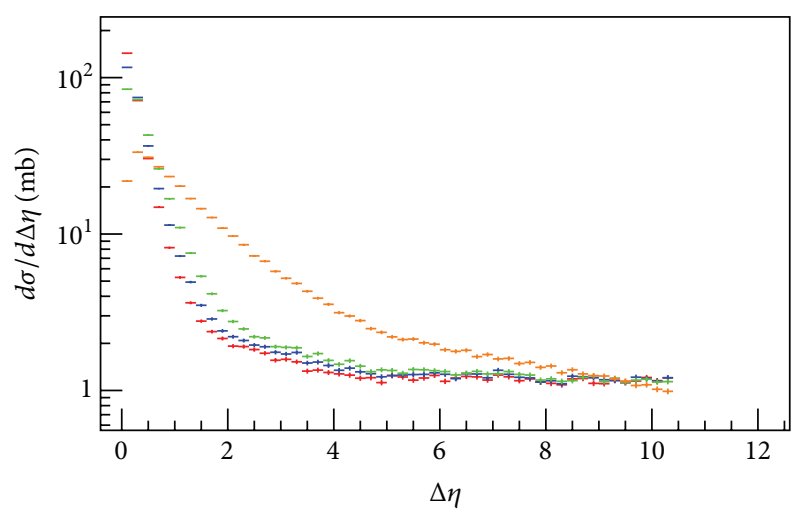

- MinBias, edge gaps, no $p_{\mathrm{T}}$ cut

- MinBias, edge gaps, $p_{\mathrm{T}}>100 \mathrm{MeV}$

MinBias, edge gaps, $p_{\mathrm{T}}>200 \mathrm{MeV}$

MinBias, edge gaps, $p_{\mathrm{T}}>500 \mathrm{MeV}$

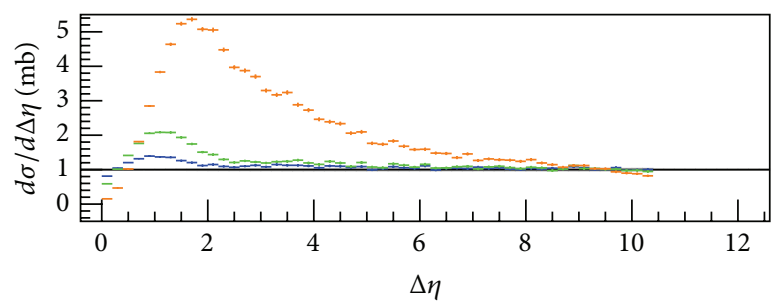

MinBias, edge gaps, $p_{\mathrm{T}}>100 \mathrm{MeV}$

- MinBias, edge gaps, $p_{\mathrm{T}}>200 \mathrm{MeV}$

MinBias, edge gaps, $p_{\mathrm{T}}>500 \mathrm{MeV}$

FIGURE 5: Large rapidity gap distribution for minimum bias events $(\mathrm{SDD}+\mathrm{DDD}+\mathrm{ND})$. The gap is defined as an edge gap from all final state particles in $|\eta|<5.2$. The "MinBias, edge gaps, no $p_{\mathrm{T}}$ cut" is used as a reference on the ratio plot.

TABLE 2: Visible cross sections for different $\Delta \eta$ cuts. A cut of $p_{\mathrm{T}}>$ $200 \mathrm{MeV}$ is applied for all final state particles in $|\eta|<5.2$ and the gap is defined as edge gap.

\begin{tabular}{lcccc}
\hline$\sigma_{\text {Process }}(\mathrm{mb})$ & $\Delta \eta>2.5$ & $\Delta \eta>3.0$ & $\Delta \eta>3.5$ & $\Delta \eta>4.0$ \\
\hline$\sigma_{\mathrm{SDD}}$ & 6.13 & 5.62 & 5.16 & 4.72 \\
$\sigma_{\mathrm{DDD}}$ & 4.48 & 4.10 & 3.72 & 3.37 \\
$\sigma_{\mathrm{SDD}+\mathrm{DDD}}$ & 10.61 & 9.72 & 8.88 & 8.09 \\
$\sigma_{\mathrm{ND}}$ & 0.22 & 0.11 & 0.05 & 0.03 \\
$\sigma_{\text {MinBias }}$ & 10.83 & 9.83 & 8.93 & 8.12 \\
\hline
\end{tabular}

with increasing $\Delta \eta$ cut. If a cut of $p_{\mathrm{T}}>200 \mathrm{MeV}$ is applied for all final state particles in $|\eta|<5.2$ and the events with an edge gap $\Delta \eta>3$ are selected, $98.8 \%$ of the minimum bias events will be diffractive dissociation events. However, with these cuts, one cannot distinguish SDD and DDD events since $42.1 \%$ of the diffractive dissociation events will be DDD events. These results are presented by a histogram in Figure 8.

4.3.2. Central Gaps. A similar event selection is performed by requiring a central gap in the events. These events should be dominated by DDD events where both diffraction systems 

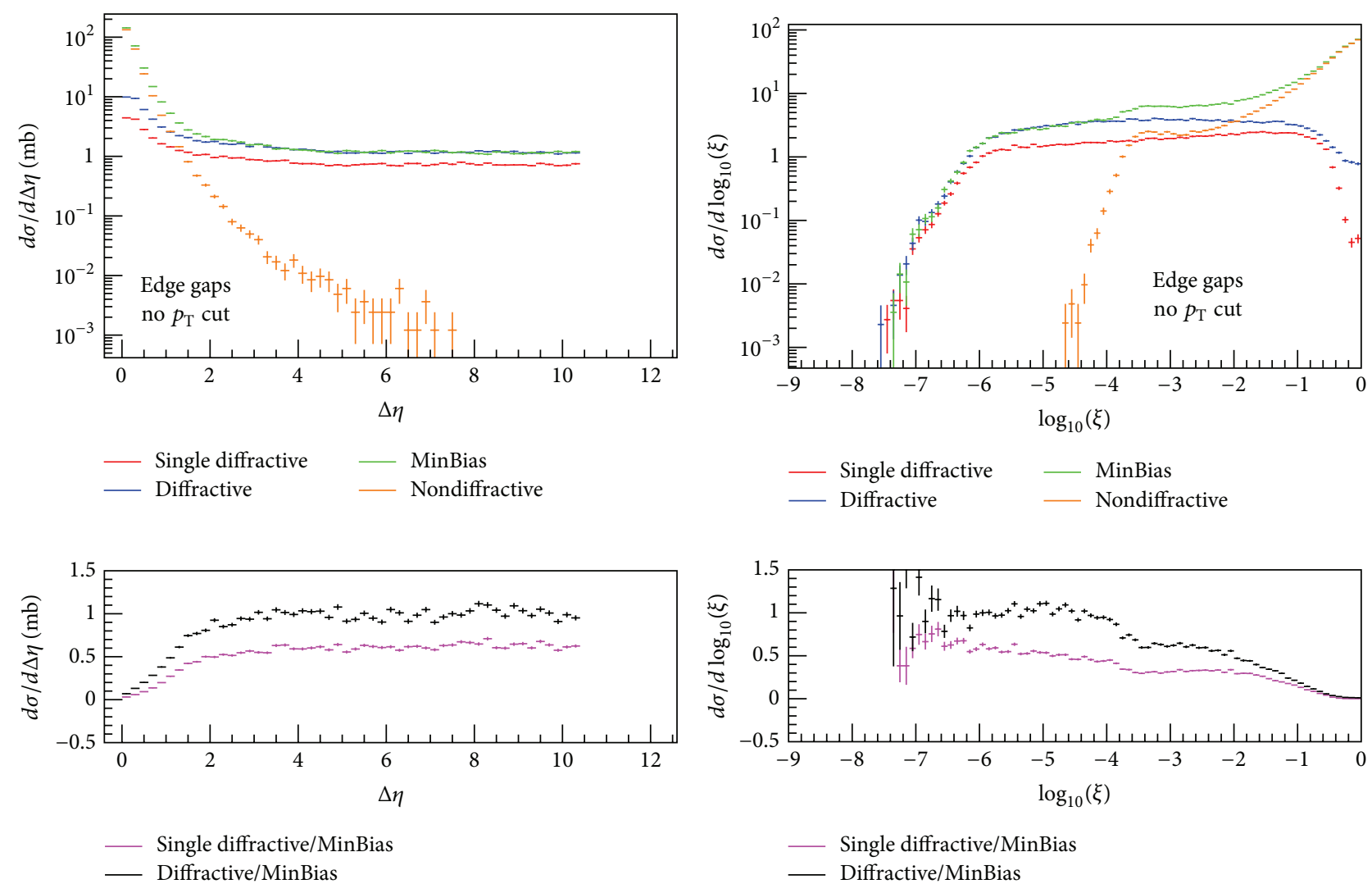

(a)
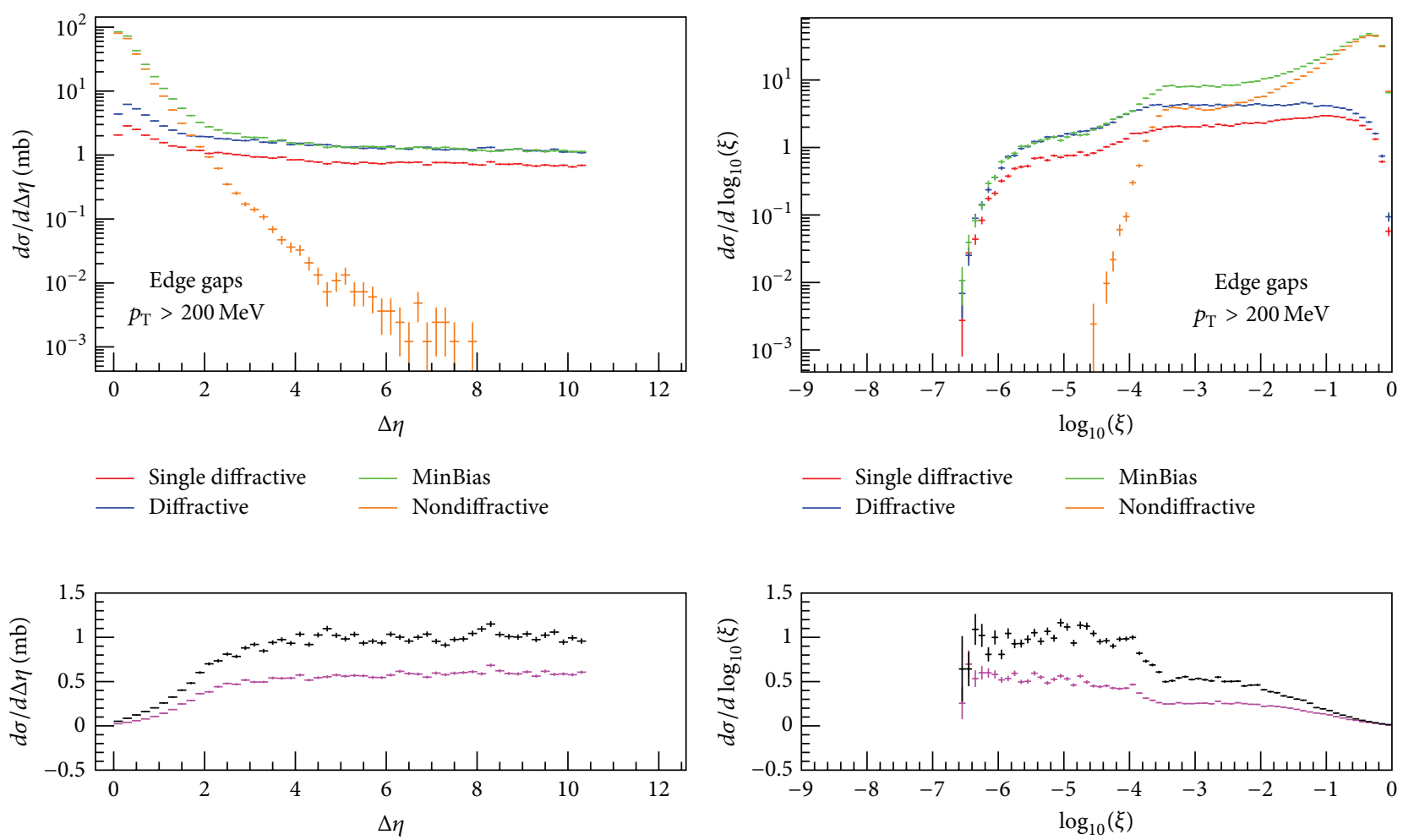

_ Single diffractive/MinBias

Diffractive/MinBias

(c)

(d)

Figure 6: Continued. 

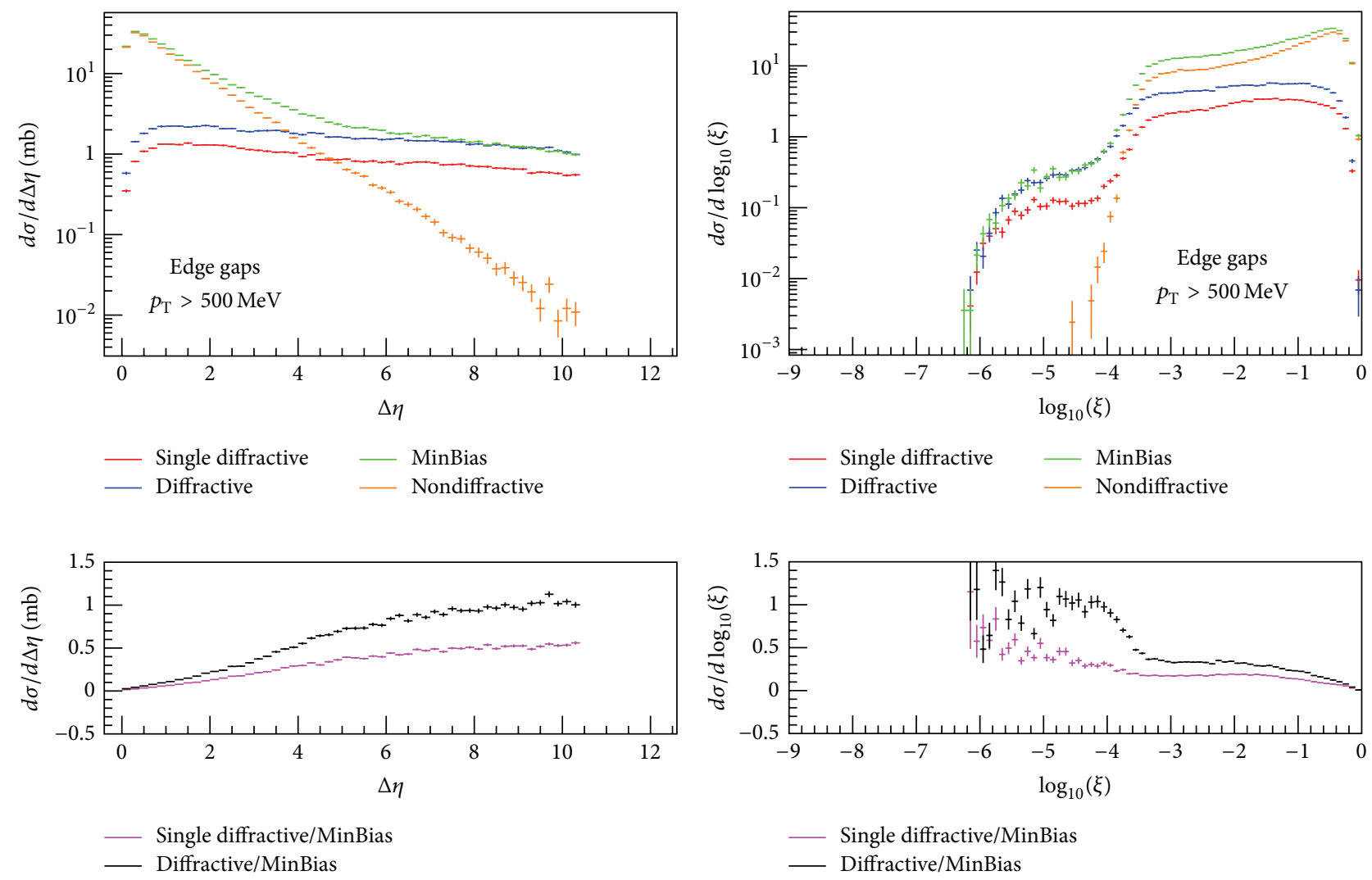

(e)

(f)

Figure 6: On the left side $d \sigma / d \Delta \eta$ and on the right side $d \sigma / d \log _{10} \xi$ for different event classes. Gap is defined as edge gap and no $p_{\mathrm{T}}$ cut (a, b), $p_{\mathrm{T}}>200 \mathrm{MeV}(\mathrm{c}, \mathrm{d})$, and $p_{\mathrm{T}}>500 \mathrm{MeV}(\mathrm{e}, \mathrm{f})$ cuts are applied for all final state particles in $|\eta|<5.2$.

TABLE 3: Visible cross sections for different cuts on the transverse momentum of the final state particles in $|\eta|<5.2$ for $\Delta \eta>3$ cut. Gap is defined as edge gap.

\begin{tabular}{|c|c|c|c|c|}
\hline$\sigma_{\text {Process }}(\mathrm{mb})$ & No $p_{\mathrm{T}}$ cut & $p_{\mathrm{T}}>100 \mathrm{MeV}$ & $p_{\mathrm{T}}>200 \mathrm{MeV}$ & $p_{\mathrm{T}}>500 \mathrm{MeV}$ \\
\hline$\sigma_{\mathrm{SDD}}$ & 5.52 & 5.60 & 5.62 & 5.80 \\
\hline$\sigma_{\mathrm{DDD}}$ & 3.42 & 3.79 & 4.10 & 5.14 \\
\hline$\sigma_{\mathrm{SDD}+\mathrm{DDD}}$ & 8.94 & 9.39 & 9.72 & 10.94 \\
\hline$\sigma_{\mathrm{ND}}$ & 0.03 & 0.06 & 0.11 & 4.38 \\
\hline$\sigma_{\text {MinBias }}$ & 8.97 & 9.45 & 9.83 & 15.32 \\
\hline
\end{tabular}

are in the calorimeter acceptance. The visible cross sections of events that pass the various $\Delta \eta$ cuts with $p_{\mathrm{T}}>200 \mathrm{MeV}$ for all final state particles are given in Table 4 . The visible cross sections for different cuts on the transverse momentum of the final state particles for $\Delta \eta>4$ are presented in Table 5. As indicated in the tables, the visible cross section decreases with increasing $\Delta \eta$. The ND contribution in minimum bias data is dominant in the larger $p_{\mathrm{T}}$ and, also, with the increasing $p_{\mathrm{T}}$ cut, visible cross section for SDD events increases while it decreases for DDD events. If a cut of $p_{\mathrm{T}}>200 \mathrm{MeV}$ is applied for all final state particles in $|\eta|<5.2$ and the events with a central gap $\Delta \eta>4$ are selected, $95.3 \%$ of the minimum bias events will be diffractive dissociation events. SDD events in this diffractive dissociation event content will be almost
TABLE 4: Visible cross sections for different $\Delta \eta$ cuts. A cut of $p_{\mathrm{T}}>$ $200 \mathrm{MeV}$ is applied for all final state particles in $|\eta|<5.2$ and the gap is defined as central gap.

\begin{tabular}{lcccc}
\hline$\sigma_{\text {Process }}(\mathrm{mb})$ & $\Delta \eta>2.5$ & $\Delta \eta>3.0$ & $\Delta \eta>3.5$ & $\Delta \eta>4.0$ \\
\hline$\sigma_{\mathrm{SDD}}$ & 0.08 & 0.03 & 0.01 & 0.003 \\
$\sigma_{\mathrm{DDD}}$ & 1.15 & 1.04 & 0.93 & 0.82 \\
$\sigma_{\mathrm{SDD}+\mathrm{DDD}}$ & 1.23 & 1.07 & 0.94 & 0.82 \\
$\sigma_{\mathrm{ND}}$ & 0.66 & 0.22 & 0.08 & 0.04 \\
$\sigma_{\text {MinBias }}$ & 1.89 & 1.29 & 1.02 & 0.86 \\
\hline
\end{tabular}

completely suppressed. These results are summarized by a histogram in Figure 9. 

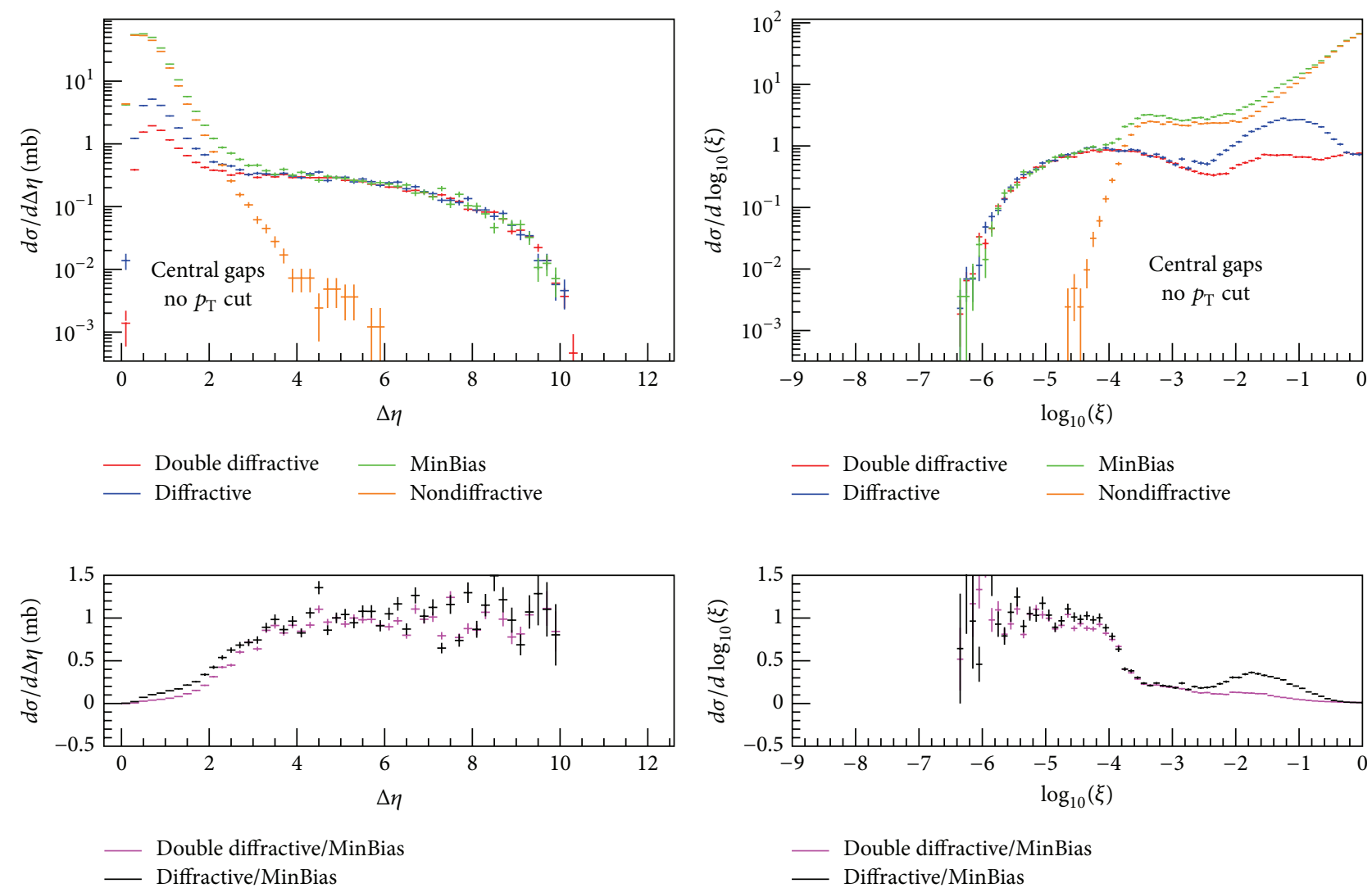

(a)
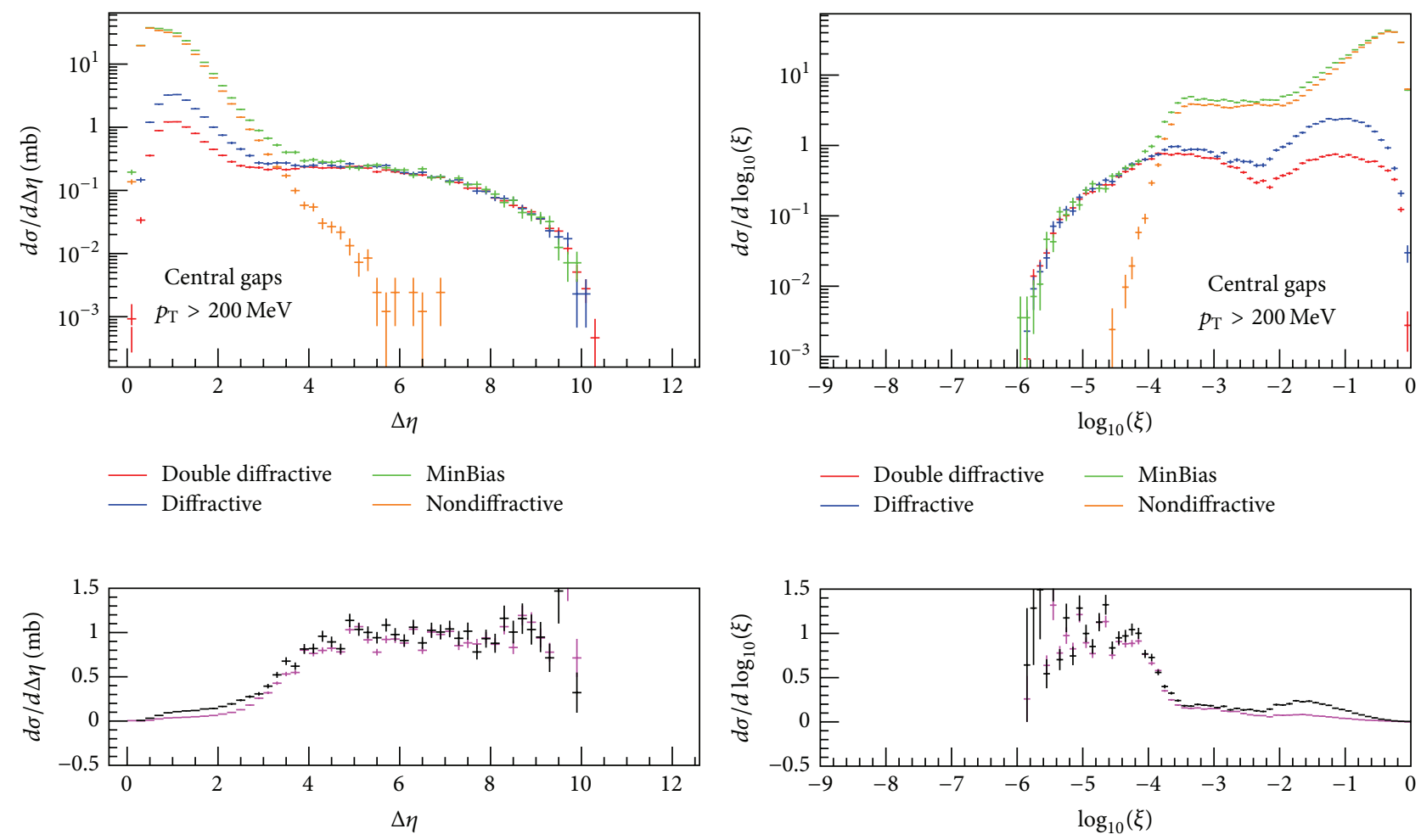

Double diffractive/MinBias Diffractive/MinBias

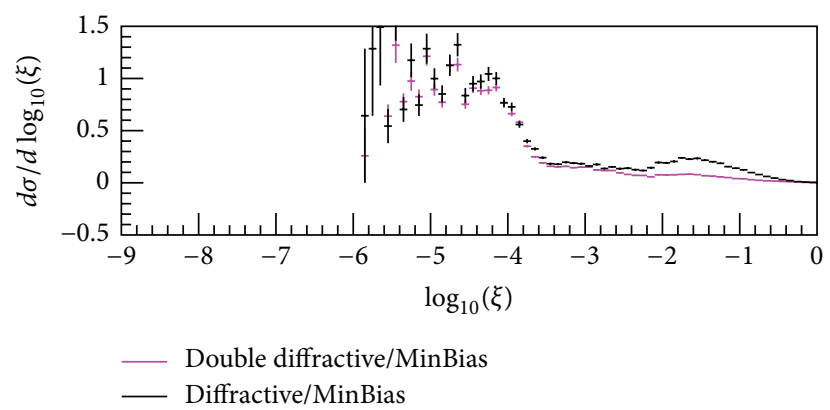

(c)

Figure 7: Continued. 

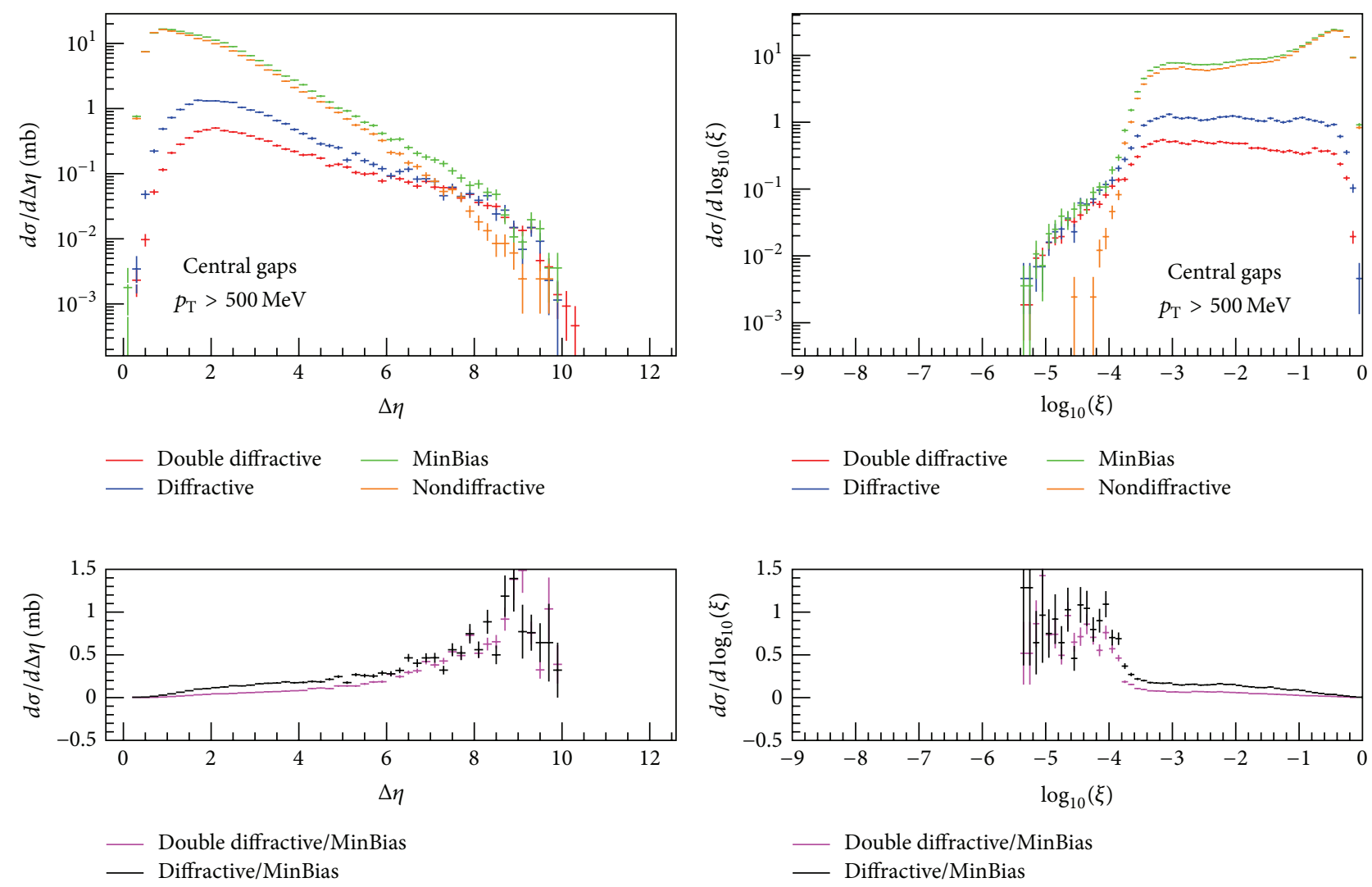

(e)

(f)

FIGURE 7: On the left side $d \sigma / d \Delta \eta$ and on the right side $d \sigma / d \log _{10} \xi$ for different event classes. Gap is defined as central gap and no $p_{\mathrm{T}}$ cut $(\mathrm{a}, \mathrm{b}), p_{\mathrm{T}}>200 \mathrm{MeV}(\mathrm{c}, \mathrm{d})$, and $p_{\mathrm{T}}>500 \mathrm{MeV}(\mathrm{e}, \mathrm{f})$ cuts are applied for all final state particles in $|\eta|<5.2$.

TABLE 5: Visible cross sections for different cuts on the transverse momentum of the final state particles in $|\eta|<5.2$ for $\Delta \eta>4$ cut. Gap is defined as central gap.

\begin{tabular}{lcccc}
\hline$\sigma_{\text {Process }}(\mathrm{mb})$ & No $p_{\mathrm{T}}$ cut & $p_{\mathrm{T}}>100 \mathrm{MeV}$ & $p_{\mathrm{T}}>200 \mathrm{MeV}$ & 0.003 \\
\hline$\sigma_{\mathrm{SDD}}$ & 0.002 & 0.001 & 0.82 & 0.18 \\
$\sigma_{\mathrm{DDD}}$ & 0.95 & 0.92 & 0.82 & 0.44 \\
$\sigma_{\mathrm{SDD}+\mathrm{DDD}}$ & 0.95 & 0.92 & 0.04 & 0.62 \\
$\sigma_{\mathrm{ND}}$ & 0.007 & 0.01 & 0.86 & 1.99 \\
$\sigma_{\text {MinBias }}$ & 0.96 & 0.93 & & 2.61 \\
\hline
\end{tabular}

Although central gaps look like an ideal cut to separate SDD and DDD events, only a small fraction of the DDD cross section has a central gap. It mostly has an edge gap. Due to a class of DDD events with a low diffractive mass on one side, the particles beyond the acceptance of the detector are not detected and they look like SDD events in the limited detector rapidity acceptance. The fraction of DDD events which can be tagged as SDD events in the limited rapidity range, $|\eta|<5.2$, is calculated. Results for different cuts on the size of the edge gap $\Delta \eta$ are given in Table 6 . As indicated in the table, for $\Delta \eta>$ $3,44.1 \%$ of the DDD events can be tagged as SDD events in the limited detector rapidity coverage. This event fraction is smaller for the larger gap sizes.
4.3.3. Multiplicity and Total Energy Deposition. In order to distinguish SDD events from DDD events with a low diffractive mass, the distributions $\sum\left(E \pm p_{z}\right)$, total energy deposition, and particle multiplicity were investigated. The sum for $\sum\left(E \pm p_{z}\right)$ runs over all final state particles in $|\eta|<5.2$. For $\mathbf{p}$ much larger than the particle mass, the longitudinal momentum $p_{z}$ is calculated as $E \cos \theta$, where $E$ is the energy of the particle and $\theta$ is the angle between the particle momentum $\mathbf{p}$ and the beam axis. The distributions for the events which have an edge gap $\Delta \eta>3$ with a cut of $p_{\mathrm{T}}>200 \mathrm{MeV}$ for all final state particles are given in Figure 10. As shown in the figure, the shapes of the distributions for different event classes look very similar 

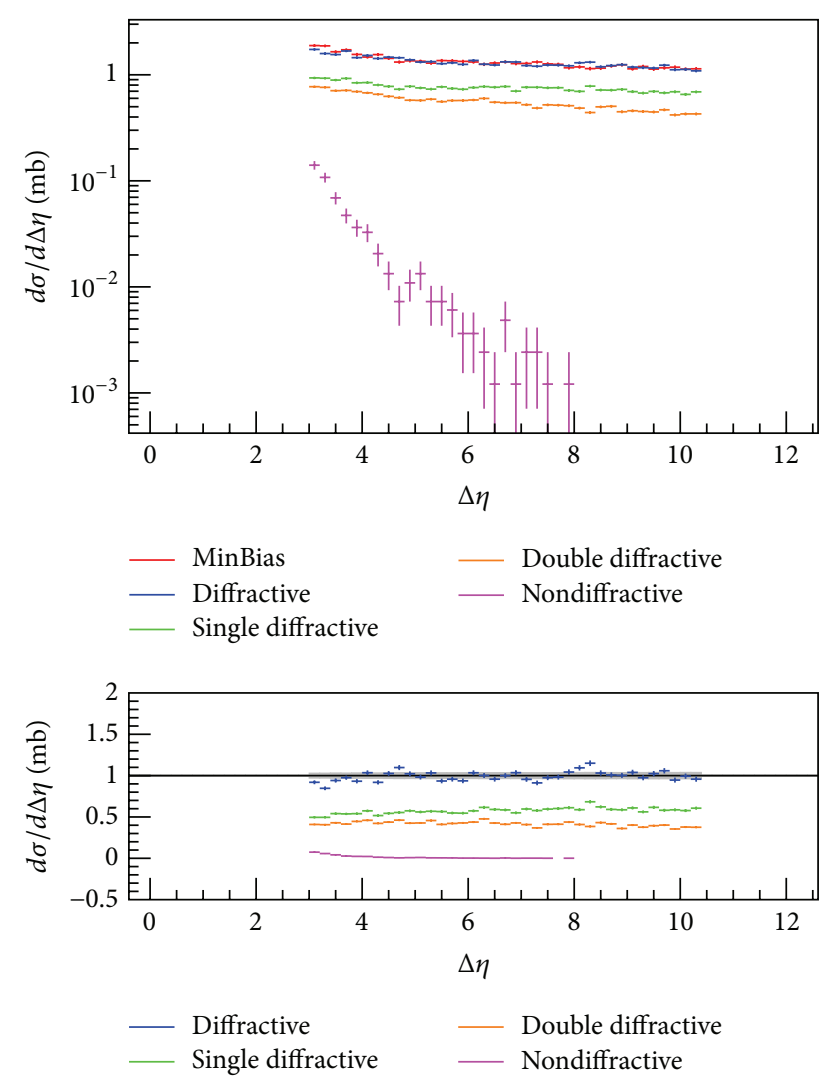

FIGURE 8: Large rapidity gap distribution for the events that have an edge gap $\Delta \eta>3$ with a cut of $p_{\mathrm{T}}>200 \mathrm{MeV}$ for all final state particles in $|\eta|<5$.2. The MinBias event class is used as a reference for the ratio.

and, therefore, it seems not possible to separate SDD from DDD events with these cuts by using edge gaps. The distributions for ND events class are not represented since there are very few ND events which pass the event selection.

The same distributions were studied also for central gaps. The distributions for the events which have a central gap $\Delta \eta>$ 4 with a cut of $p_{\mathrm{T}}>200 \mathrm{MeV}$ for all final state particles in $|\eta|<5.2$ are given in Figure 11.

The distributions for events with an edge gap do not distinguish SDD and DDD (Figure 10), and, as regards those for central gaps (Figure 11), there are some differences, but the SDD contribution is anyway very suppressed in these events.

4.4. Events Tagging at Very Forward Rapidities. As it was discussed in the previous sections, a class of DDD events with a low diffractive mass on one side can be tagged as SDD events in the limited rapidity acceptance of the detector. Since the particles in such events dissociate into the forward rapidities, looking at the particle activity in the very forward detectors can provide more accurate information about the type of the process. The ATLAS and CMS Zero Degree Calorimeters, ZDC, are located 140 meters away from the interaction point (IP) on both sides, at the end of the straight LHC beam-line section $[16,17]$. The ZDC cover
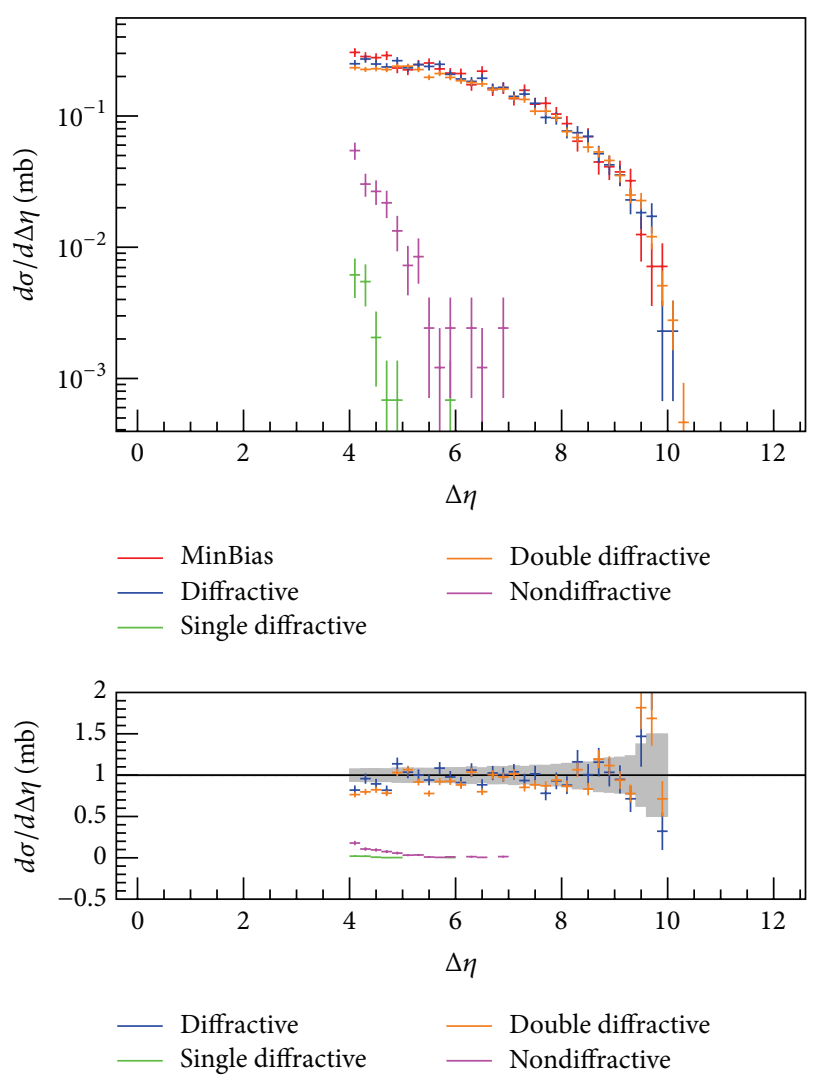

FIGURE 9: Large rapidity gap distribution for the events that have a central gap $\Delta \eta>4$ with a cut of $p_{\mathrm{T}}>200 \mathrm{MeV}$ for all final state particles in $|\eta|<5$.2. The MinBias event class is used as a reference for the ratio.

the pseudorapidity region $|\eta|>8.1$ and are able to detect very forward neutral particles $\left(n, \gamma, \pi^{\circ}\right)$ at a $0^{\circ}$ polar angle.

The total energy deposition and the multiplicity of the neutral particles in the ZDC acceptance are studied, in order to investigate whether there is a way to separate SDD and lowmass DDD events. An edge gap was required in the events with a gap size $\Delta \eta>3$ and with a cut of $p_{\mathrm{T}}>200 \mathrm{MeV}$ for all final state particles in $|\eta|<5$.2. Additionally, a certain amount of energy deposition $E \neq 0$ was required in the opposite side from the gap (at either $\eta<0$ or $\eta>0$ depending on the gap position). In Figure 12, the total energy deposition and the multiplicity of the neutral particles in $\mathrm{ZDC}^{\prime}$ (the ZDC detector on the side opposite the gap) and ZDC" (the ZDC detector on the side with the gap) are given with a cut of $100 \mathrm{MeV}$ for the transverse momentum of the neutral particles $\left(p_{\mathrm{T}}^{0}\right)$. The $p_{\mathrm{T}}^{0}>100 \mathrm{MeV}$ is a reasonable cut given the ZDC noise levels. Also, the fraction of the events which have at least one neutral particle in $\mathrm{ZDC}$ " for different cuts on the transverse momentum of the neutral particles with different cuts on the size of the gap is given in Table 7 for SDD and in Table 8 for DDD processes. As can be seen, for the events that have an edge gap with a size of $\Delta \eta>3$ and $p_{\mathrm{T}}^{0}>100 \mathrm{MeV}, \mathrm{SDD}$ events have almost no neutral particle in $\mathrm{ZDC}^{\prime \prime}$ while $60.2 \%$ of the DDD events have at least one neutral particle with $p_{\mathrm{T}}^{0}>100 \mathrm{MeV}$ scattering into 

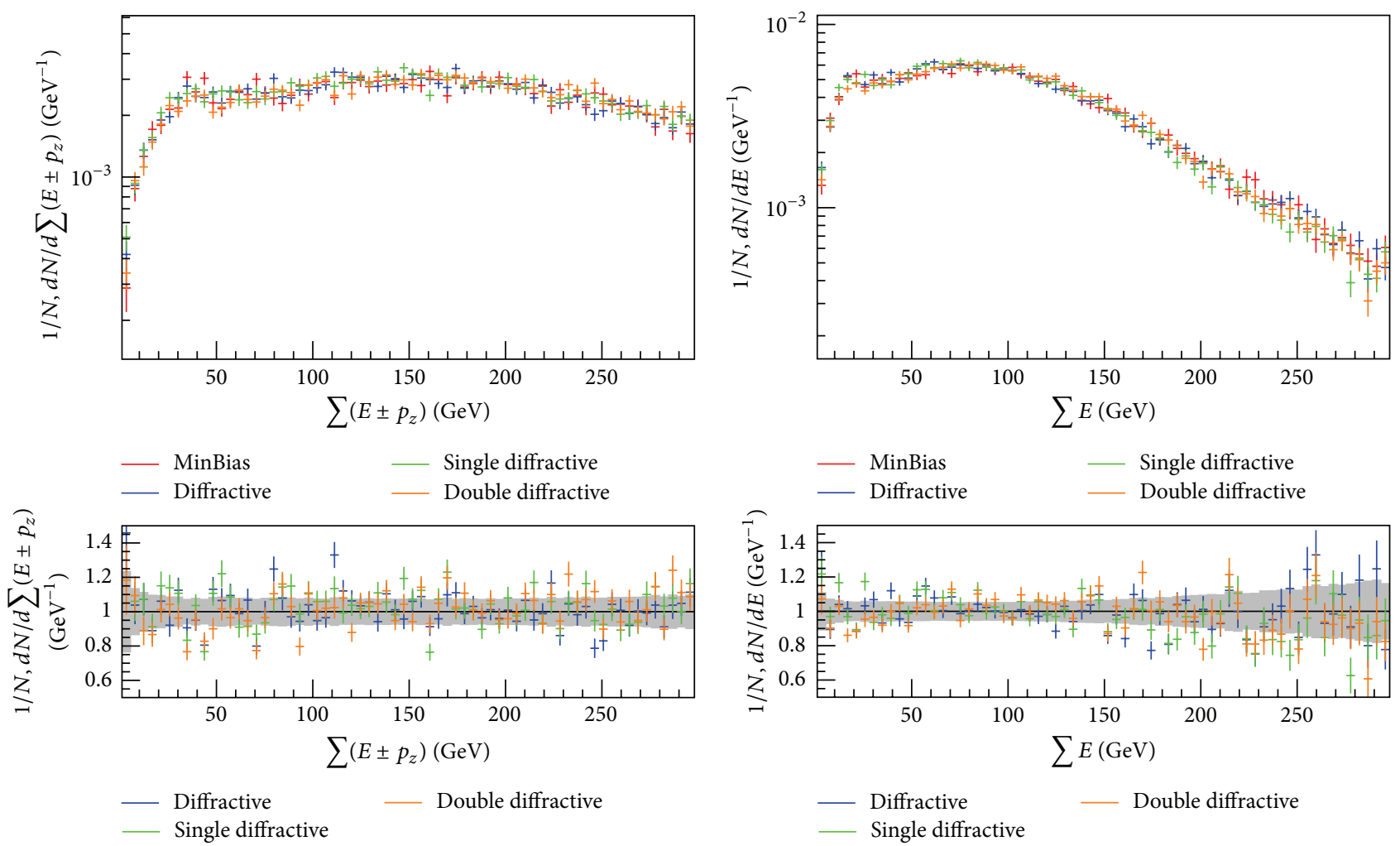

(a)

(b)
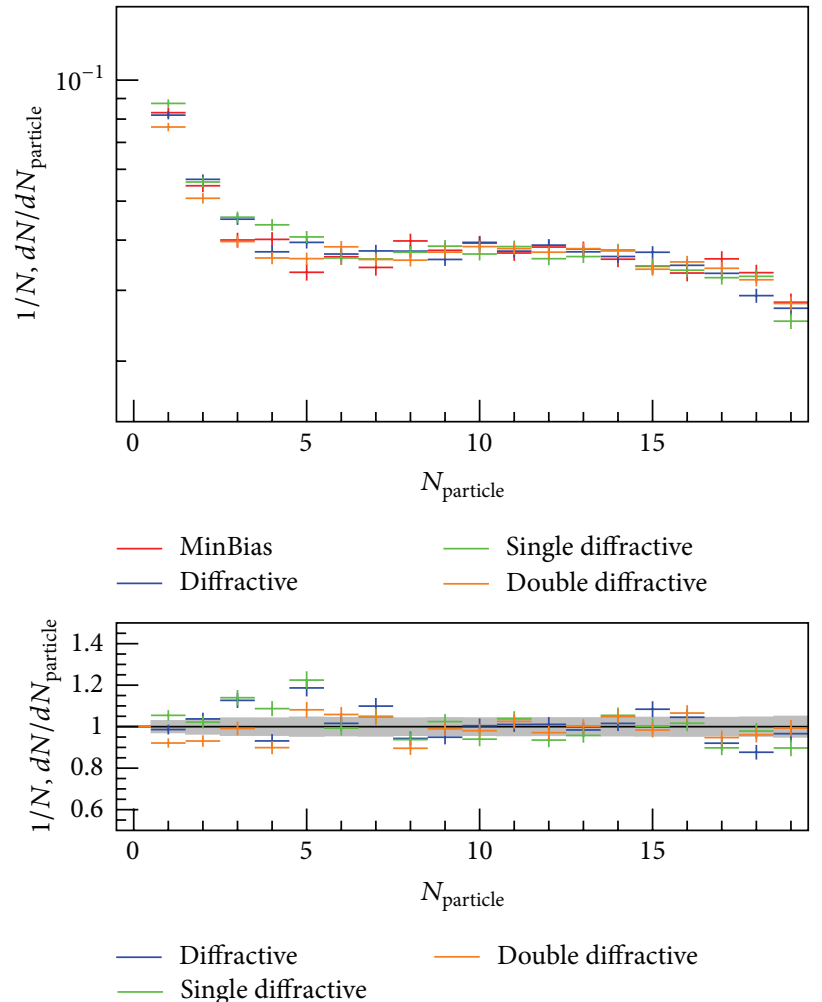

(c)

Figure 10: Distributions of $\sum\left(E \pm p_{z}\right)(\mathrm{a})$, total energy deposition (b), and particle multiplicity (c) for the events that have an edge gap $\Delta \eta>3$ with a $p_{\mathrm{T}}>200 \mathrm{MeV}$ cut on final state particles in $|\eta|<5$.2. The distributions are normalized to the number of events, $N$, that pass the analysis cuts. The MinBias event class is used as a reference for the ratio. 

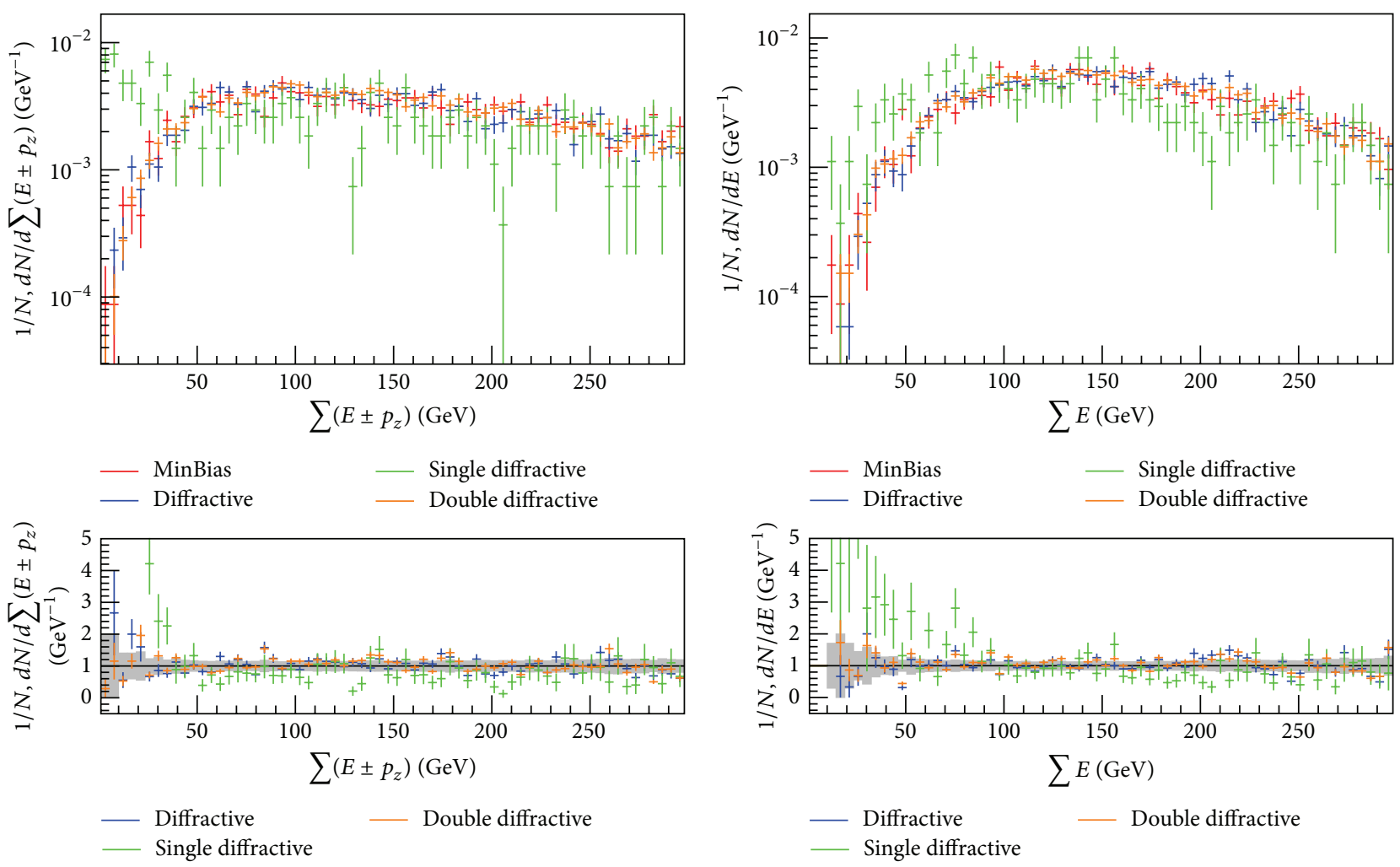

(a)

(b)

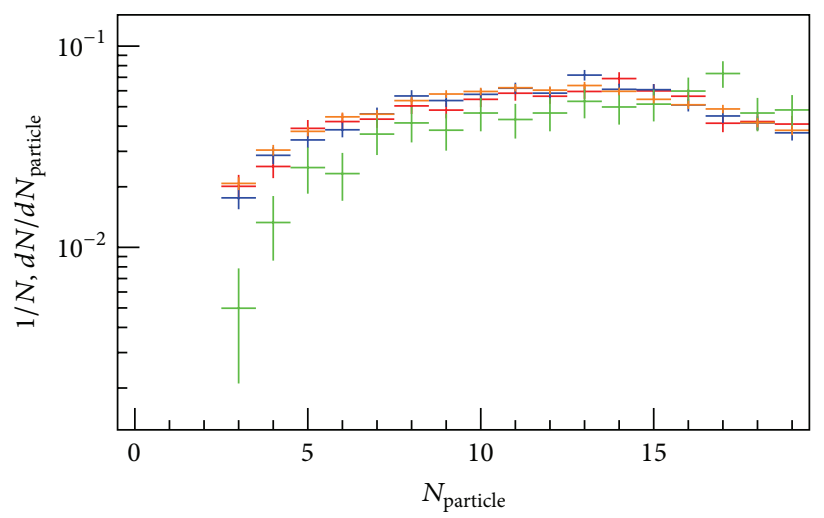

$\begin{array}{ll}\text { MinBias } & - \text { Single diffractive } \\ \text { Diffractive } & \text { Double diffractive }\end{array}$

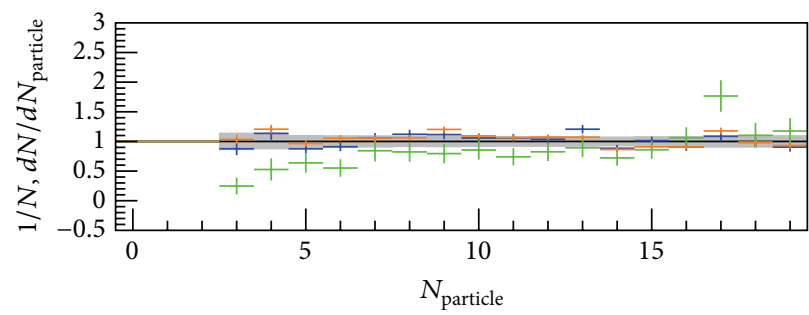

Diffractive
Single diffractive

(c)

FIGURE 11: Distributions of $\sum\left(E \pm p_{z}\right)(\mathrm{a})$, total energy deposition (b), and particle multiplicity (c) for the events that have a central gap $\Delta \eta>4$ with a $p_{\mathrm{T}}>200 \mathrm{MeV}$ cut on final state particles in $|\eta|<5.2$. The distributions are normalized to the number of events, $N$, that pass the analysis cuts. The MinBias event class is used as a reference for the ratio. 


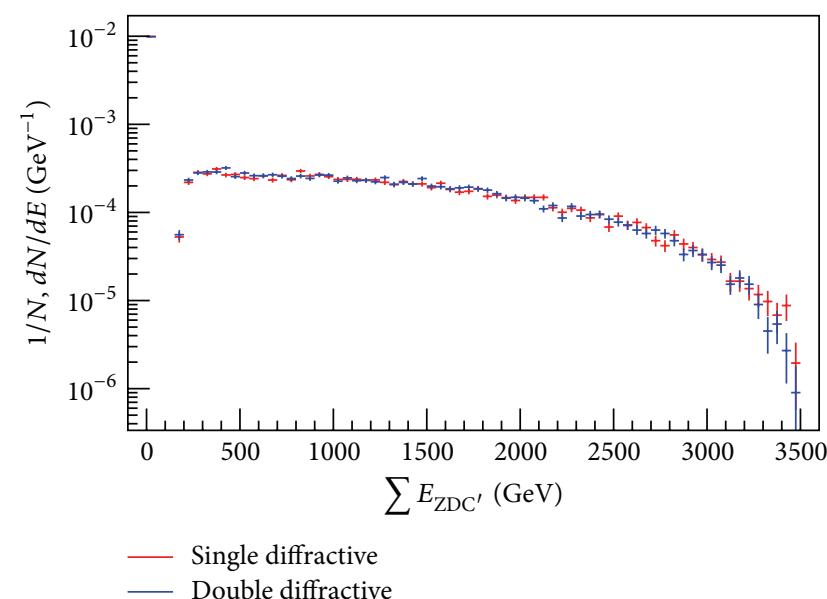

(a)

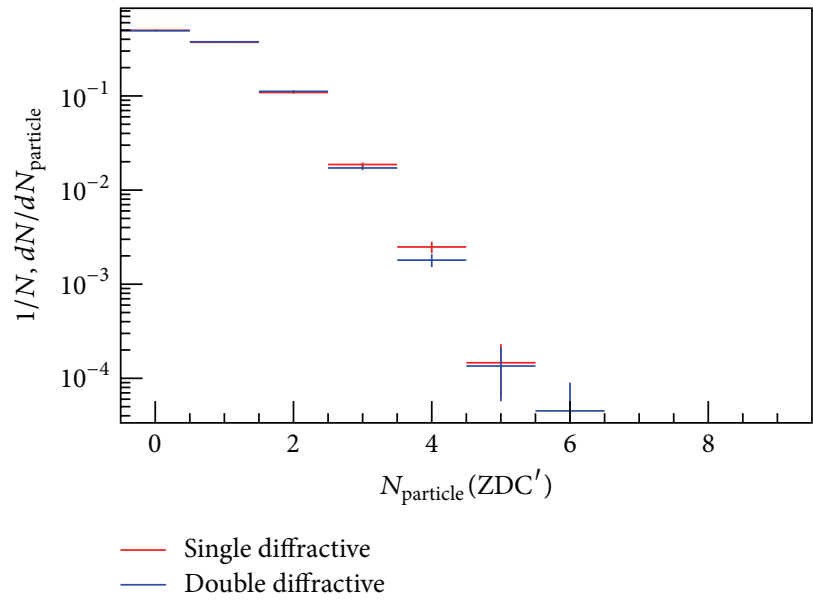

(c)

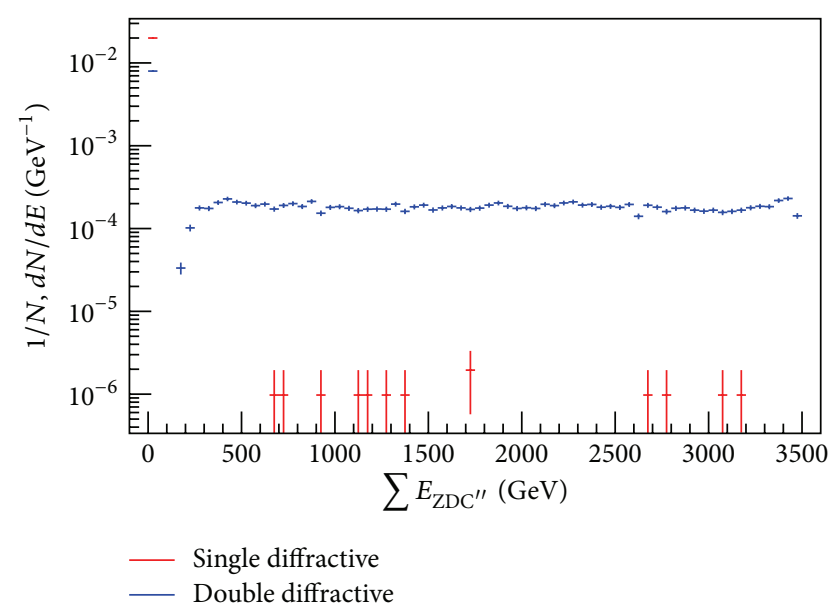

(b)

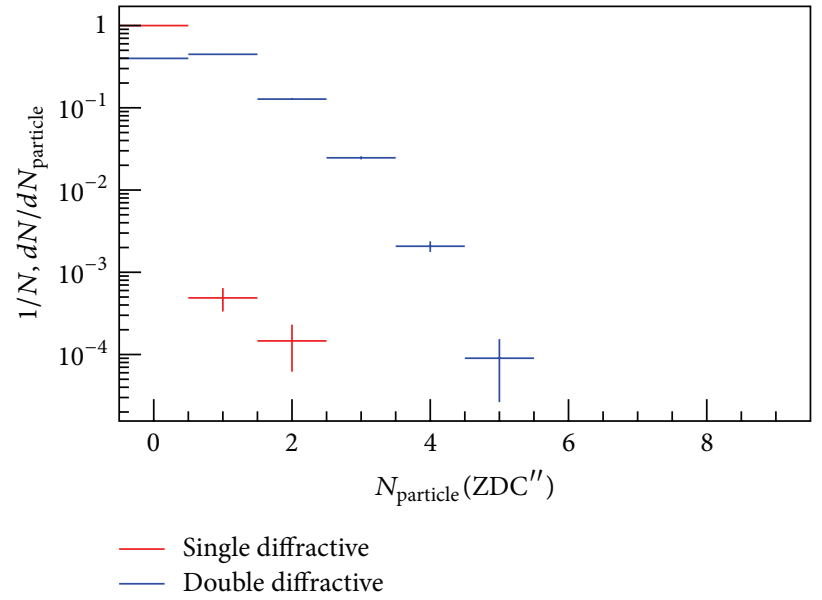

(d)

FIGURE 12: The total energy deposition and the multiplicity of the neutral particles with $p_{\mathrm{T}}^{0}>100 \mathrm{MeV}$ in the ZDC detectors for the events that have an edge gap in $|\eta|<5.2$ with a gap size $\Delta \eta>3$, and with $E \neq 0$ in the opposite side from the gap (either at $\eta<0$ or $\eta>0$ depending on the gap position). A cut of $p_{\mathrm{T}}>200 \mathrm{MeV}$ was applied for the final state particles in $|\eta|<5.2$ to find the size of the gap. ZDC' refers to the ZDC which is on the side opposite to the gap and $Z^{\prime \prime} C^{\prime \prime}$ is the ZDC on the side with the gap. Total energy deposition in (a) ZDC' and (b) $\mathrm{ZDC}^{\prime \prime}$ and particle multiplicity in (c) ZDC' and (d) ZDC ${ }^{\prime \prime}$ are given. The distributions are normalized to the number of events, $N$, that pass the analysis cuts.

TABLE 6: The fraction of DDD events which can be tagged as SDD events in the limited rapidity range $|\eta|<5.2$. Gap is defined as edge gap.

\begin{tabular}{lcccc}
\hline Event class & $\Delta \eta>2.5$ & $\Delta \eta>3.0$ & $\Delta \eta>3.5$ & $\Delta \eta>4.0$ \\
\hline DDD & $48.2 \%$ & $44.1 \%$ & $40.2 \%$ & $36.3 \%$ \\
\hline
\end{tabular}

these forward rapidities. Although ZDC can provide a better distinction for SDD and DDD events, about $40 \%$ of the DDD events do not have any particles within $\mathrm{ZDC}^{\prime \prime}$ and, therefore, they cannot be distinguished from SDD events in $|\eta|<5.2$. The selection efficiency can be improved by extending the nominal rapidity coverage of detectors.

4.5. Bias from Vertex. One of the background sources of diffractive processes at the LHC is the radiation coming from
TABLE 7: The fraction of SDD events which have at least one neutral particle in ZDC" (the ZDC detector on the side with the gap) is given for different cuts on the size of the $\Delta \eta$ and with different thresholds for the transverse momentum of the neutral particles. The gap is defined as an edge gap and the final state particles within $|\eta|<5.2$ with $p_{\mathrm{T}}>200 \mathrm{MeV}$ are used to find the size of the gap.

\begin{tabular}{lcccc}
\hline & $\Delta \eta>2.5$ & $\Delta \eta>3.0$ & $\Delta \eta>3.5$ & $\Delta \eta>4.0$ \\
\hline No $p_{\mathrm{T}}^{0}$ cut & $0.23 \%$ & $0.10 \%$ & $0.06 \%$ & $0.03 \%$ \\
$p_{\mathrm{T}}^{0}>100 \mathrm{MeV}$ & $0.20 \%$ & $0.09 \%$ & $0.05 \%$ & $0.03 \%$ \\
$p_{\mathrm{T}}^{0}>200 \mathrm{MeV}$ & $0.16 \%$ & $0.08 \%$ & $0.05 \%$ & $0.02 \%$ \\
\hline
\end{tabular}

noncolliding bunches. The common practice to eliminate such background is usually to require a primary vertex in the events. The primary vertex is defined as the location of pp collision. The number of tracks, or theoretically number 

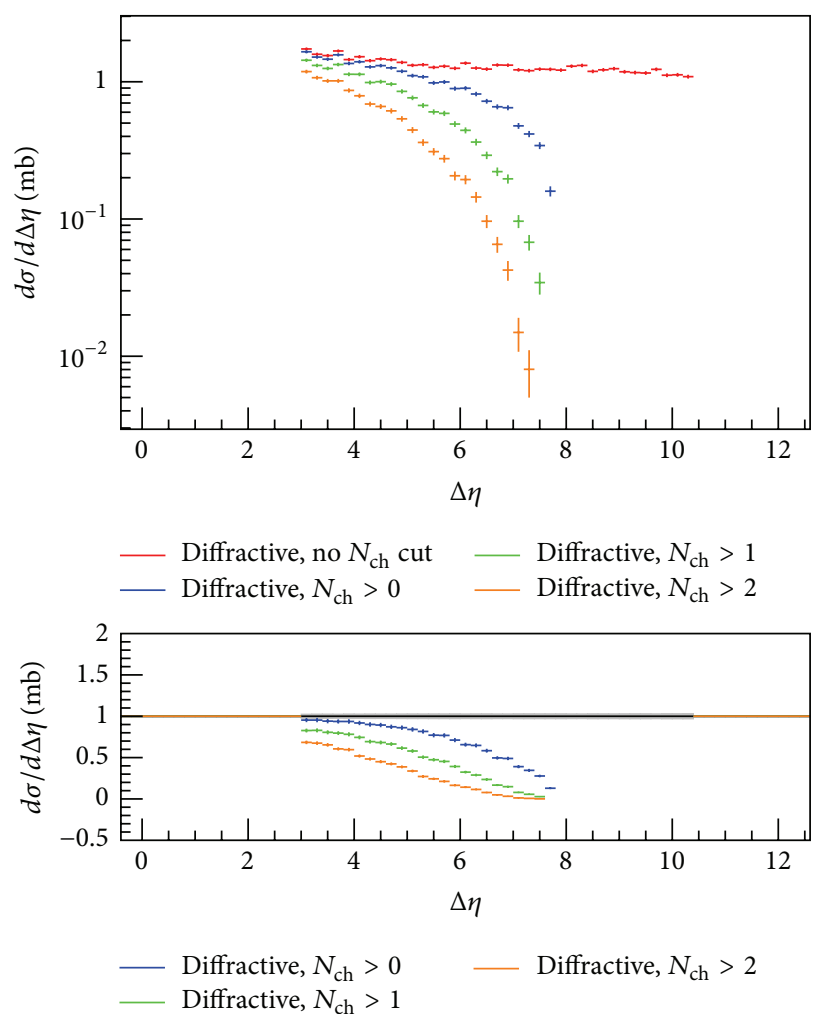

FIGURE 13: Diffractive dissociation events without a primary vertex and with a primary vertex for the different number of charged particles, $N_{\mathrm{ch}}$. Tracker region is considered as $|\eta|<2.5$, and $p_{\mathrm{T}}>200 \mathrm{MeV}$ charged particles are used to form the vertex. Only the events that have an edge gap $\Delta \eta>3$ with a cut of $p_{\mathrm{T}}>200 \mathrm{MeV}$ for all final state particles in $|\eta|<5.2$ are considered. The vertex requirement suppresses the events that have $\Delta \eta>8$ which corresponds to the very low-mass soft diffractive processes.

TABLE 8: The fraction of DDD events which have at least one neutral particle in ZDC" (the ZDC detector on the side with the gap) is given for different cuts on the size of $\Delta \eta$ and with different thresholds for the transverse momentum of the neutral particles. The gap is defined as an edge gap and the final state particles within $|\eta|<5.2$ with $p_{\mathrm{T}}>$ $200 \mathrm{MeV}$ are used to find the size of the gap.

\begin{tabular}{lcccc}
\hline & $\Delta \eta>2.5$ & $\Delta \eta>3.0$ & $\Delta \eta>3.5$ & $\Delta \eta>4.0$ \\
\hline No $p_{\mathrm{T}}^{0}$ cut & $68.93 \%$ & $68.90 \%$ & $68.93 \%$ & $68.97 \%$ \\
$p_{\mathrm{T}}^{0}>100 \mathrm{MeV}$ & $60.28 \%$ & $60.20 \%$ & $60.18 \%$ & $60.22 \%$ \\
$p_{\mathrm{T}}^{0}>200 \mathrm{MeV}$ & $47.27 \%$ & $47.09 \%$ & $46.89 \%$ & $46.76 \%$ \\
\hline
\end{tabular}

of charged particles, associated with a primary vertex can be different for different processes. In some cases, such as low-mass diffractive dissociation, the system can dissociate into the very forward rapidities and thus all the particles may appear at small polar angles. The problem in this case is the limited detector instruments in this region. The tracker of ATLAS and CMS, which is used to measure charged particles, covers the pseudorapidity region $|\eta|<2.5$. Therefore, it will not be possible to measure charged particles when the system dissociates into the very forward rapidities. These types of events may not form a reconstructable primary vertex if all the particles are outside of the tracker region.

Figure 13 shows the large rapidity gap distribution for diffractive events with and without a primary vertex where
TABLE 9: The fraction of events without a primary vertex and with a primary vertex for the different number of charged particles, $N_{\mathrm{ch}}$. Tracker region is considered as $|\eta|<2.5$ and $p_{\mathrm{T}}>200 \mathrm{MeV}$ charged particles are used to form the vertex. Only the events that have an edge gap $\Delta \eta>3$ with a cut of $p_{\mathrm{T}}>200 \mathrm{MeV}$ for all final state particles in $|\eta|<5.2$ are considered.

\begin{tabular}{lcccc}
\hline Event class & No $N_{\mathrm{ch}}$ cut & $N_{\mathrm{ch}}>0$ & $N_{\mathrm{ch}}>1$ & $N_{\mathrm{ch}}>2$ \\
\hline $\begin{array}{l}\text { Single } \\
\text { diffractive }\end{array}$ & $41.1 \%$ & $19.9 \%$ & $13.1 \%$ & $8.5 \%$ \\
$\begin{array}{l}\text { Double } \\
\text { diffractive }\end{array}$ & $44.3 \%$ & $23.0 \%$ & $15.4 \%$ & $10.1 \%$ \\
$\begin{array}{l}\text { Diffractive } \\
\text { MinBias }\end{array}$ & $42.4 \%$ & $21.1 \%$ & $14.1 \%$ & $9.2 \%$ \\
Nondiffractive & $13.8 \%$ & $7.0 \%$ & $4.7 \%$ & $3.1 \%$ \\
\hline
\end{tabular}

the tracker region is considered as $|\eta|<2.5$ and $p_{\mathrm{T}}>$ $200 \mathrm{MeV}$ charged particles are used to form the vertex. The fraction of events that pass the cut, for different number of charged particles, $N_{\text {ch }}$, is given in Table 9 for different event classes. Requiring a primary vertex which is reconstructed with two or more charged particles suppresses at least $33.2 \%$ of the diffractive events. Particularly, very low-mass soft diffractive dissociation events with a very large gap, $\Delta \eta>$ 8 , are suppressed by the primary vertex cut. Therefore, instead of a primary vertex cut, one should investigate other 


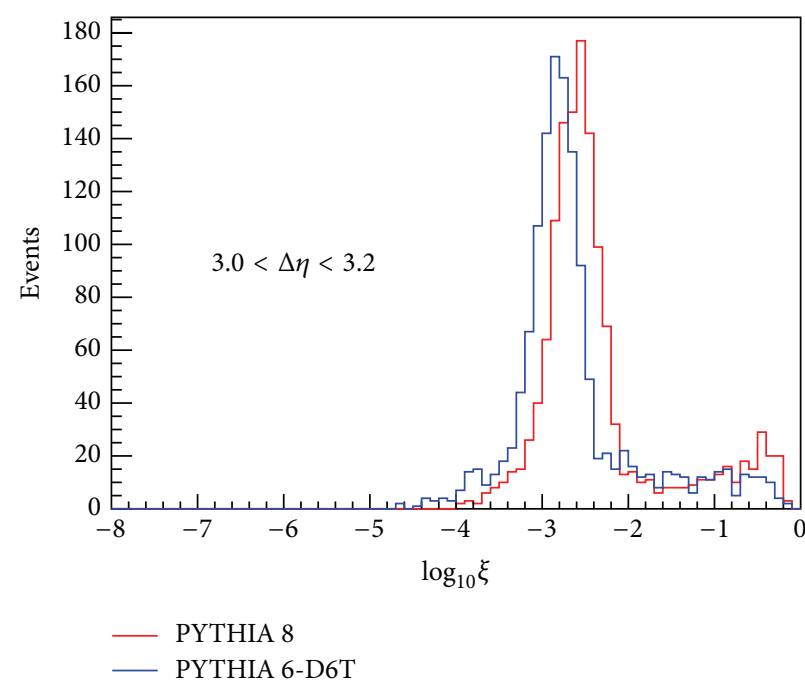

(a)

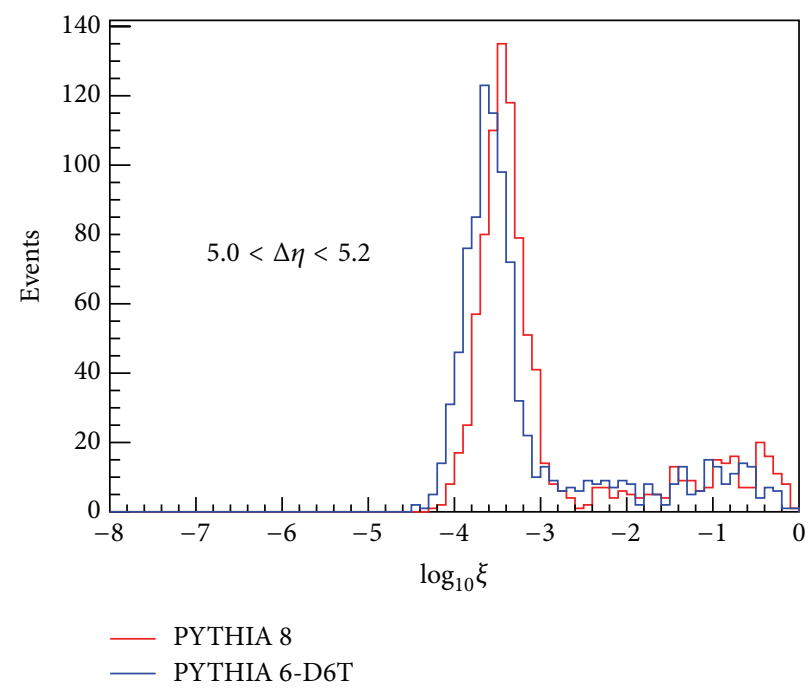

(b)

FIGURE 14: The range of $\xi$ values for SDD events with a gap size (a) $3.0<\Delta \eta<3.2$ and (b) $5.0<\Delta \eta<5.2$. Events are simulated by PYTHIA 8 and PYTHIA 6-D6T, and only edge gaps are considered with a cut of $p_{\mathrm{T}}>200 \mathrm{MeV}$ for the final state particles in $|\eta|<5.2$.

experimental ways of eliminating the background coming from noncolliding bunches. In this limited tracker range, a primary vertex cut is not practical to perform measurements for the diffractive dissociation events.

4.6. Model Dependency. As it was discussed in the previous sections, a calculation of the diffractive mass can be made through its relation to the size of the rapidity gap. However, this is model dependent. For a given gap size, the range of $\xi$ value can be different for different models. As an example, two different sizes of edge gap, $3.0<\Delta \eta<3.2$ and $5.0<$ $\Delta \eta<5.2$, are considered and the diffractive masses of the dissociation systems are calculated for SDD events. The events are simulated by PYTHIA 8 and PYTHIA 6-D6T [18], which use different set of parameters for the simulation. The range of $\xi$ values and the difference in the range between models are given in Figure 14.

A study of the model dependence of correcting an inclusive minimum bias measurement to one for SDD processes only is presented in Figure 15. It looks like the difference is quite small especially in the $\Delta \eta>3$ region, but of course the difference could be larger with other models. The correction to get to SDD is large (about a factor of 2) and it is preferable to measure $\Delta \eta$ distribution without performing such a correction, such that dependence on MC models is minimized.

\section{Conclusions}

Methods to select soft diffraction dissociation at the LHC experiments ATLAS and CMS are studied by using large rapidity gaps in the events. It is shown that the larger the rapidity covered is, the more precisely the measurements for diffractive dissociation events can be done. A primary vertex requirement in the event selection is not practical due to the limited tracker range $|\eta|<2$.5. In particular, very low-mass soft diffractive events which dissociate into the very forward rapidities and have a gap with a size of $\Delta \eta>8$ are suppressed by the primary vertex requirement.

In the limited detector rapidity coverage, $|\eta|<5.2$, one can select a sample of events of which $98.8 \%$ are diffractive dissociation, according to PYTHIA 8 , by requiring an edge gap with a gap size $\Delta \eta>3$ and with a cut of $p_{\mathrm{T}}>200 \mathrm{MeV}$ for all final state particles. However, with this event selection, $42.1 \%$ of the diffractive dissociation events will be DDD events and it seems not possible to fully separate SDD from DDD events by edge gap reconstruction in $|\eta|<5$.2. Central gaps look like a better candidate in order to distinguish SDD and DDD events; however, only a small fraction of DDD events have a central gap. Using Zero Degree Calorimeters, a more accurate distinction between SDD and DDD events can be done up to a certain degree. This study also puts forward the importance of ZDC in diffractive measurements.

\section{Competing Interests}

The authors declare that they have no competing interests.

\section{Acknowledgments}

Valuable discussions with Hannes Jung are thankfully acknowledged. Sercan Sen would like to thank University College London HEP group members for their kind hospitality. This work was supported by the Marie Curie Research Training Network "MCnet" (Contract no. MRTN- 

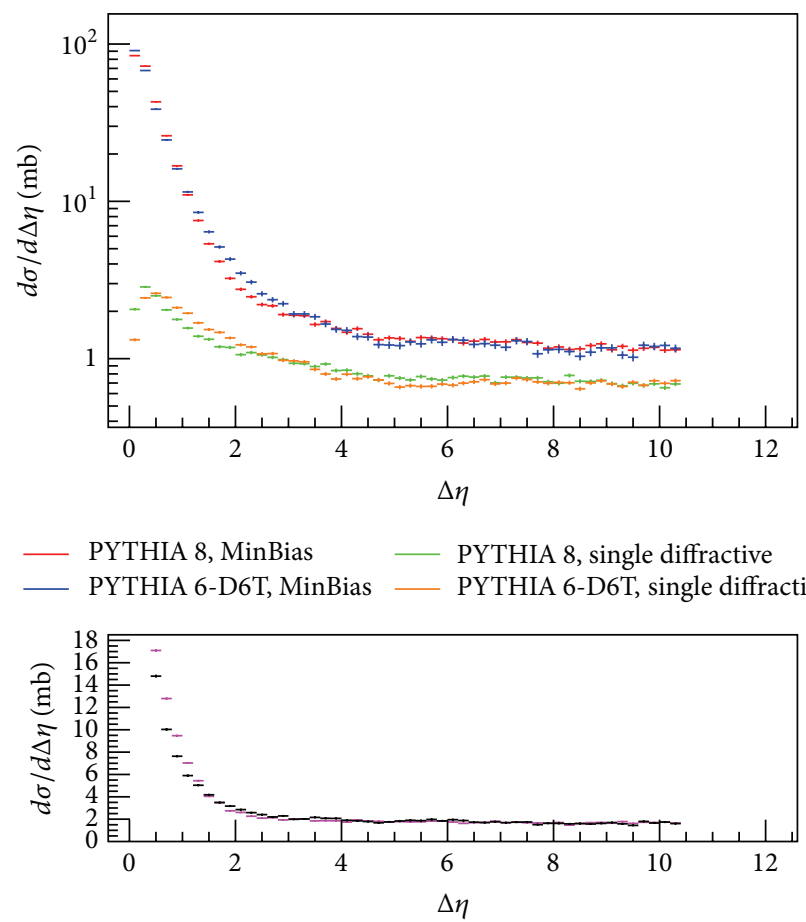

— PYTHIA 8, MinBias/single diffractive
— PYTHIA 6-D6T, MinBias/single diffractive

(a)
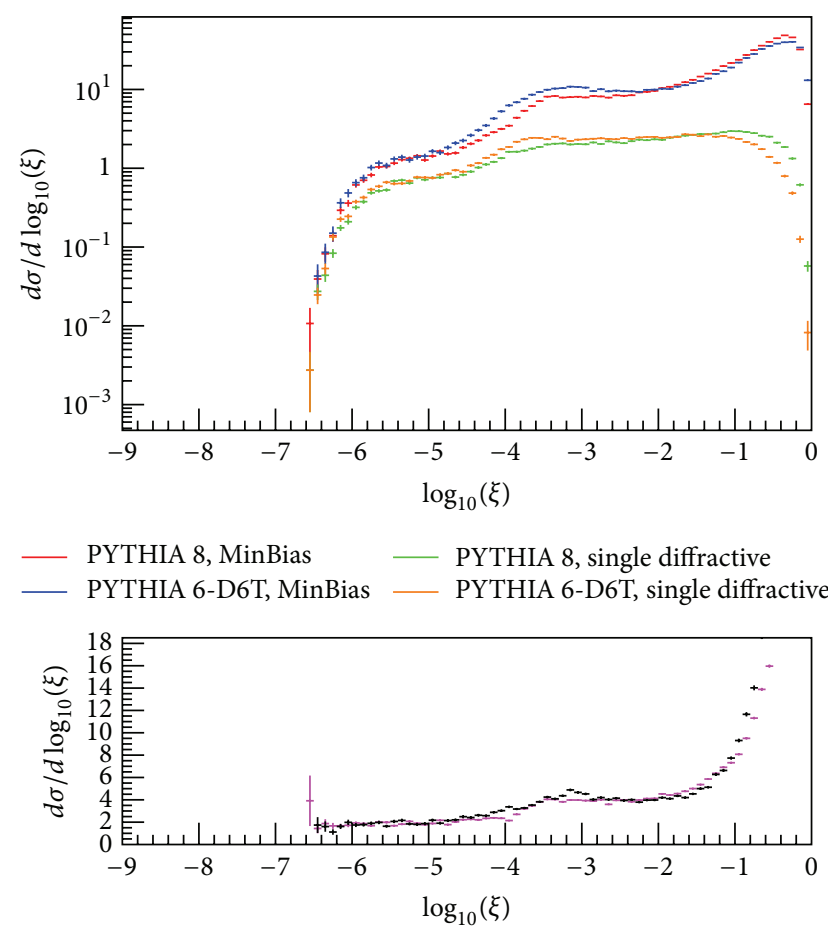

PYTHIA 8, MinBias/single diffractive - PYTHIA 6-D6T, MinBias/single diffractive

(b)

FIGURE 15: The distribution of $d \sigma / d \Delta \eta$ (a) and $d \sigma / d \log _{10} \xi$ (b) for different event classes simulated by PYTHIA 8 and PYTHIA 6-D6T. Only edge gaps are considered with a cut of $p_{\mathrm{T}}>200 \mathrm{MeV}$ for all final state particles in $|\eta|<5.2$.

CT-2006-035606) and by Hacettepe University, Scientific Research Projects Program through Project 014 A 602 006824.

\section{References}

[1] M. Albrow, A. De Roeckb, V. A. Khoze et al., "Forward physics with rapidity gaps at the LHC," Journal of Instrumentation, vol. 4, 2009.

[2] V. A. Khoze, F. Krauss, A. D. Martin, M. G. Ryskin, and K. C. Zapp, "Diffraction and correlations at the LHC: definitions and observables," The European Physical Journal C, vol. 69, no. 1, pp. 85-93, 2010.

[3] G. Aad, E. Abat, J. Abdallah et al., "The ATLAS experiment at the CERN Large Hadron Collider," Journal of Instrumentation, vol. 3, no. 8, Article ID S08003, 2008.

[4] S. Chatrchyan, G. Hmayakyan, V. Khachatryan et al., "The CMS experiment at the CERN LHC," Journal of Instrumentation, vol. 3, no. 8, Article ID S08004, 2008.

[5] S. Donnachie, H. G. Dosch, O. Nachtmann, and P. Landshoff, Pomeron Physics and QCD, vol. 19 of Cambridge Monographs on Particle Physics, Nuclear Physics and Cosmology, Cambridge University Press, 2002.

[6] V. Barone, High-Energy Particle Diffraction, Springer, Berlin, Germany, 2002.

[7] M. G. Ryskin, A. D. Martin, V. A. Khoze, and A. G. Shuvaev, "Soft physics at the LHC", Journal of Physics G: Nuclear and Particle Physics, vol. 36, no. 9, Article ID 093001, 2009.
[8] B. Abelev, J. Adam, D. Adamová et al., "Measurement of inelastic, single- and double-diffraction cross sections in protonproton collisions at the LHC with ALICE," The European Physical Journal C, vol. 73, no. 6, article 2456, pp. 1-20, 2013.

[9] G. Antchev, P. Aspell, I. Atanassov et al., "Measurement of proton-proton elastic scattering and total cross-section at $\sqrt{s}=$ 7 TeV," Europhysics Letters, vol. 101, no. 2, Article ID 21002, 2013.

[10] G. Aad, B. Abbott, J. Abdallah et al., "Rapidity gap cross sections measured with the ATLAS detector in $p p$ collisions at $\sqrt{s}=$ 7 TeV," The European Physical Journal C, vol. 72, article 1926, 2012.

[11] V. Khachatryan, A. M. Sirunyan, A. Tumasyan et al., "Measurement of diffractive dissociation cross sections in $p p$ collisions at $\sqrt{s}=7$ TeV,' Physical Review D, vol. 92, no. 1, Article ID 012003, 32 pages, 2015.

[12] T. Sjöstrand, S. Mrenna, and P. Z. Skands, "A brief introduction to PYTHIA 8.1," Computer Physics Communications, vol. 178, no. 11, pp. 852-867, 2008.

[13] F. Abe, M. Albrow, D. Amidei et al., "Observation of rapidity gaps in $\bar{p} p$ collisions at $1.8 \mathrm{TeV}$," Physical Review Letters, vol. 74, no. 6, pp. 855-859, 1995.

[14] D. Acosta, T. Affolder, H. Akimoto et al., "Central pseudorapidity gaps in events with a leading antiproton at the Fermilab Tevatron $\bar{p} p$ collider," Physical Review Letters, vol. 91, Article ID 011802, 2003.

[15] A. Buckley, J. Butterworth, D. Grellscheid et al., "Rivet user manual," Computer Physics Communications, vol. 184, no. 12, pp. 2803-2819, 2013. 
[16] O. Grachov, M. Murray, J. Wood, Y. Onel, S. Sen, and T. Yetkin, "Commissioning of the CMS zero degree calorimeter using LHC beam," Journal of Physics: Conference Series, vol. 293, Article ID 012040, 2011.

[17] S. White, “The ATLAS zero degree calorimeter," Nuclear Instruments and Methods in Physics Research Section A, vol. 617, no. 1-3, pp. 126-128, 2010.

[18] T. Sjöstrand, S. Mrenna, and P. Z. Skands, "PYTHIA 6.4 physics and manual," Journal of High Energy Physics, vol. 2006, no. 5, article 026, 2006. 

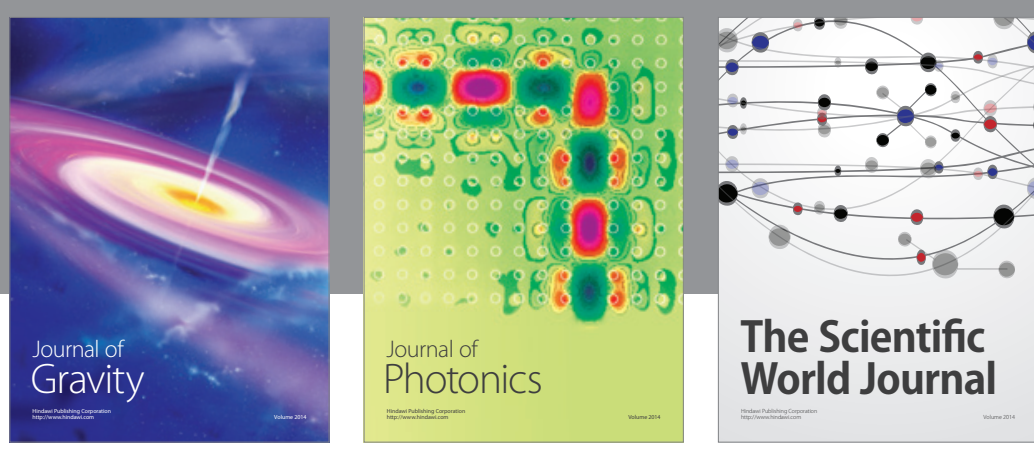

The Scientific World Journal
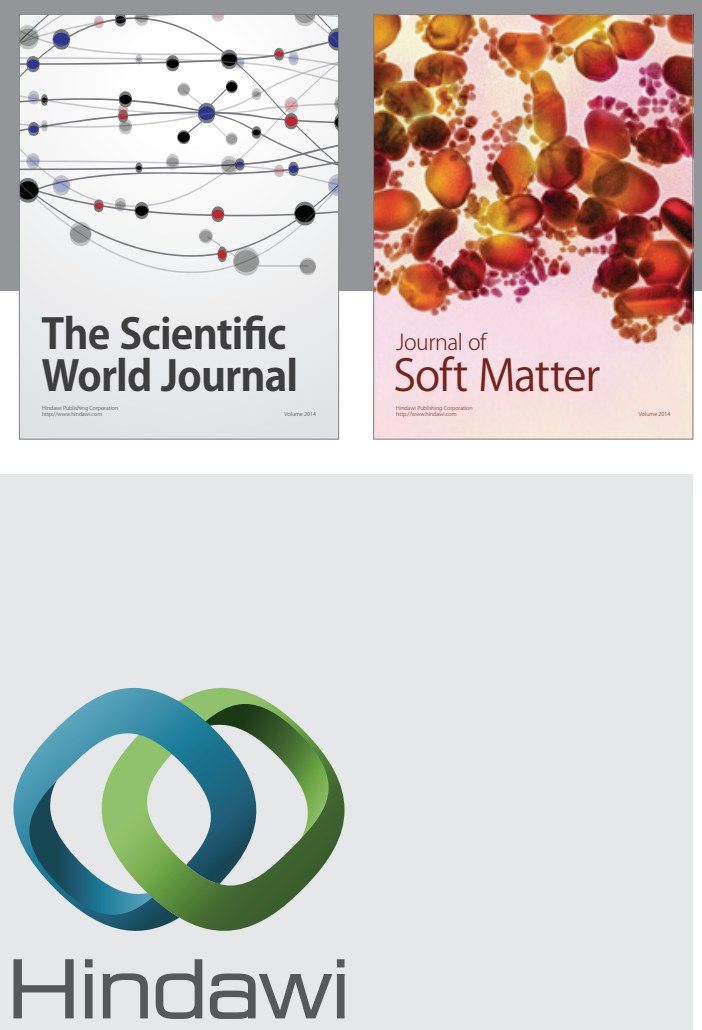

Submit your manuscripts at

http://www.hindawi.com

nternational Journal of

Statistical Mechanics
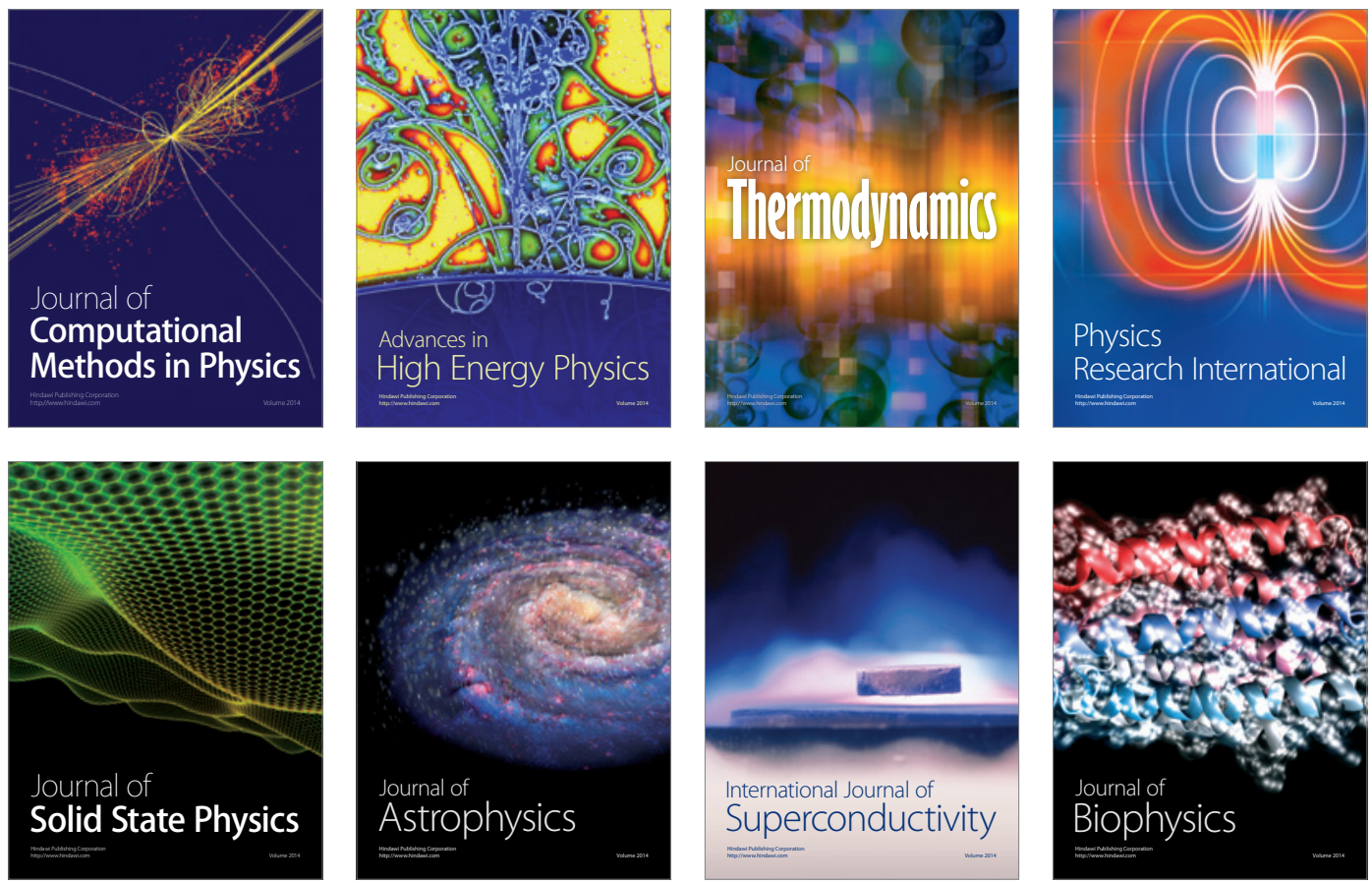
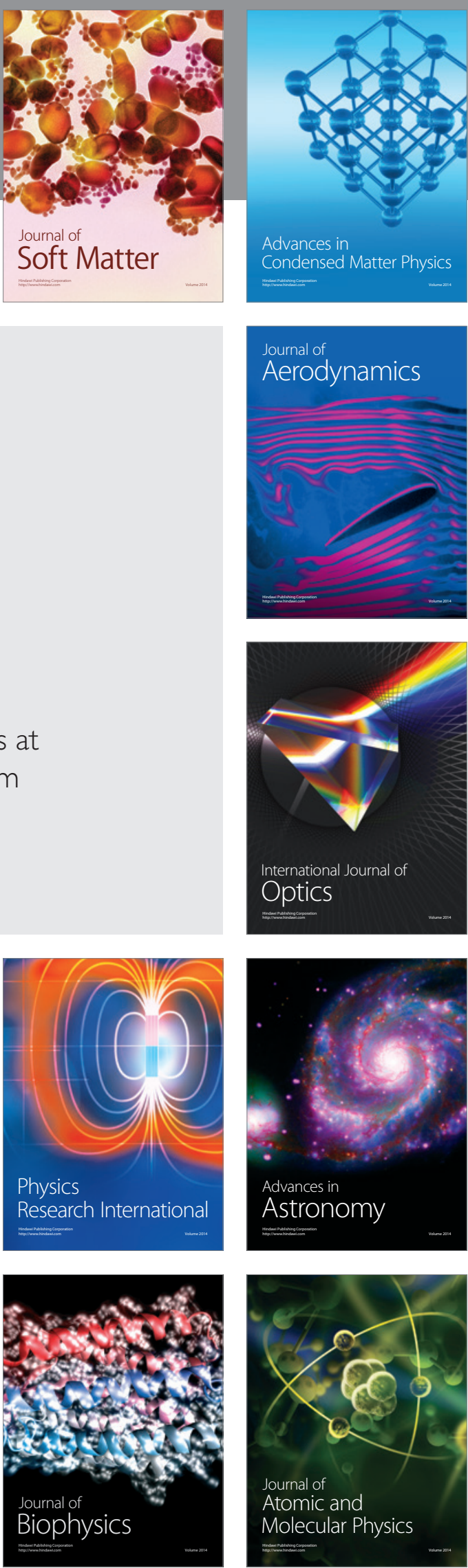